\title{
Natural formations at the Earth-Moon triangular point in perturbed restricted problems ${ }^{\text {th }}$
}

\author{
F.J.T. Salazar ${ }^{\mathrm{a}, *}$, O.C. Winter ${ }^{\mathrm{a}}$, E.E. Macau ${ }^{\mathrm{b}}$, J.J. Masdemont ${ }^{\mathrm{c}}$, G. Gómez ${ }^{\mathrm{d}}$ \\ ${ }^{a}$ UNESP-Grupo de Dinâmica Orbital e Planetologia, Guaratinguetá, SP 12516-410, Brazil \\ ${ }^{b}$ Instituto Nacional de Pesquisas Espaciais, Av. dos Astronautas 1758, São José dos Campos, SP \\ 12227-010, Brazil \\ ${ }^{c}$ Departament de Matemàtica Aplicada I, ETSEIB-UPC, Avda. Diagonal 647, 08028 Barcelona, \\ Spain \\ ${ }^{d}$ Departament de Matemàtica Aplicada i Anàlisi, UB, Gran Vía 585, 08007 Barcelona, Spain
}

\begin{abstract}
Previous studies for small formation flying dynamics about triangular libration points have determined the existence of regions of zero and minimum relative radial acceleration with respect to the nominal trajectory, that prevent from the expansion or contraction of the constellation. However, these studies only considered the gravitational force of the Earth and the Moon using the Circular Restricted Three Body Problem (CRTBP) scenario. Although the CRTBP model is a good approximation for the dynamics of spacecraft in the Earth-Moon system, the nominal trajectories around equilateral libration points are strongly affected when the primary orbit eccentricity and solar gravitational force are considered. In this manner, the goal of this work is the analysis of the best regions to place a formation that is flying close a bounded solution around $L_{4}$, taking into account the Moon's eccentricity and Sun's gravity. This model is not only more realistic for practical engineering applications but permits to determine more accurately the fuel consumption to maintain the geometry of the formation.
\end{abstract}

Keywords: Formation Flight of Satellites; Zero Relative Radial Acceleration; Earth-Moon system; Elliptic Restricted Three Body Problem; Bicircular Four Body Problem; Equilateral Libration Point

\footnotetext{
This template can be used for all publications in Advances in Space Research.

*Corresponding author. Tel.: +55 12981015572

Email addresses: e7940@hotmail.com (F.J.T. Salazar), ocwinter@gmail.com (O.C. Winter), elbert.macau@inpe.br (E.E. Macau), josep@barquins.upc.edu (J.J. Masdemont), gerard@maia.ub.es (G. Gómez)
} 


\section{Introduction}

The concept of Satellite Formation Flying (SFF) means to have two or more spacecraft in orbit such that their relative positions remain constant or obeying a certain dynamical configuration along the trajectory (Sholomitsky et al., 1977; Battrick, 2000; Bristow et al., 2000; Burns et al., 2000; Ticker and Azzolini, 2000; Fridlund and Capaccioni, 2002; Hsiao and Scheeres, 2002). This concept involves the control over the coordinated motion of a group of satellites, with the goal of maintaining a specific geometric space configuration among the elements of the formation (Sabol et al., 2001). It allows that a group of small satellites, arranged in a space formation flying, operate like a large 'virtual satellite'. This formation will have many benefits over single satellites, including simpler designs, faster build times, cheaper and unprecedented high resolution (Kapila et al., 2000).

Over the past decade, numerous formation flying missions have been conceived. In regard to outer space, the Laser Interferometer Space Antenna (LISA) is a proposed mission that uses three identical spacecraft flying in a triangular formation, with equal arms of 5 million kilometers each, to observe astrophysical and cosmological sources of gravitational waves of low frequencies (Peterseim et al., 2000). Another example is the PRISMA formation flying and rendezvous technology mission, that consists of two spacecraft: Mango and Tango, with a total mass of about $200 \mathrm{~kg}$, and its primary purpose is to demonstrate formation flying and rendezvous technology, not only in terms of Guidance, Navigation and Control software and algorithms, but also in terms of instruments and operational aspects (e.g., small rocket engines and Micro Electro Mechanical Systems). PRISMA mission was launched successfully on June 25, 2010, into a Sun synchronous orbit at approximately $750 \mathrm{~km}$ altitude (Persson et al., 2006; Gill et al., 2007; Hellman et al., 2009; Persson et al., 2010). Other interesting formation flying mission is the New Worlds Observer (NWO) (Cash et al., 2009). NWO consists of a large telescope and an occulter spacecraft in tandem at about 50,000 km apart. The two spacecraft would be flying at the Earth-Sun $L_{2}$ Lagrangian point or in a drift-away solar orbit. Its purpose is to discover and analyze terrestrial extra-solar planets. The NWO planned launch date is about 2018. On the other hand, in the planetary orbit scenario, an example of formation is the pair of satellites Landsat 7 with Earth Observing-1, mission designed to enable the development of future Earth imaging observatories that will have a significant increase in performance while reducing cost and mass (Flick, 2012).

For outer space scenario, there exists interest of major space agencies to position $\mathrm{SFF}$ in halo orbits around Lagrangian points $L_{1}$ and $L_{2}$ (Faquar, 1968; Gómez et 
al., 2001a; Howell and Marchand, 2005; Marchand and Howell, 2005; Héritier and Howell, 2014) or $L_{4}$ and $L_{5}$ (Simó et al., 1987). In the case of Earth-Moon system, $L_{4}$ and $L_{5}$ points could be excellent locations to place space telescopes for astronomical observations or a space station (Schutz, 1977). In addition, Defilippi (1977) made a review of the ideas of American physicist Gerard K. O'Neill (O'Neill, 1974) about building space colonies at the $L_{4}$ and $L_{5}$ positions. These space stations could be used as a way-point for travelling to and from the region between Earth's atmosphere and the Moon (cis-lunar space). Despite this advantage, today there are no missions orbiting $L_{4}$ or $L_{5}$ points for any celestial pair of primaries.

One of the problems of positioning satellites in formation flying is the cost to maintain them continuously orbiting each other. For the reason that keeping a formation from drifting apart and achieving mission requirements is expected to require significantly more fuel than station keeping a single spacecraft. Previous studies like those by Catlin and McLaughlin (2007), Wong (2009) and Salazar et al. (2014) on $\mathrm{SFF}$ about $L_{4}$ in the Earth-Moon system have been carried out. The motion of small formation flying near Triangular Libration points was studied adopting the Circular Restricted Three Body Problem (CRTBP) model. Catlin and McLaughlin (2007) show that formations are possible at the Triangular points for uncontrolled trajectories due to the stability of stationary solutions. On the other hand, Wong (2009) establishes that a system control is required and develops strategies for keeping a spacecraft formation system at $L_{4}$. Salazar et al. (2014) present analytical and numerical methods based on the linearization of the relative equations of motion with respect to periodic orbits around $L_{4}$ to find the regions where the radial component of the relative acceleration with respect to periodic orbits is zero, such that, large variations of the mutual distance between satellites are avoided. Therefore, supposing that the relative velocity of each satellite with respect to the periodic orbit is zero, the most feasible relative positions for formation flying have to be orthogonal to the relative acceleration. Gómez et al. (2006) applied a similar methodology for halo orbits around the collinear equilibrium point $L_{2}$ in the Sun-Earth system, which are unstable, and showing the existence of regions of zero relative radial acceleration for halo orbits.

Catlin and McLaughlin (2007), Wong (2009) and Salazar et al. (2014) show that velocity change requirements demanded by the control methods would be very small in the CRTBP scenario. Thus, these studies conclude that nonlinear aspects as well as perturbations forces (e.g. solar gravity, solar radiation pressure) are necessary to provide a more real-world accurate descriptions of motion and control of formation flying around equilateral libration points in the Earth-Moon system.

In order to cope with Moon's eccentricity and Sun's perturbation, two scenar- 
ios for formation flying are considered here. The first one is the Elliptic Restricted Three Body Problem (ERTBP). The equations of motion for the ERTBP in the pulsating synodic system are non-autonomous, but there are still five equilibrium points with similar dynamics to the ones in the CRTBP (Danby, 1964; Érdi et al., 2009). Thus, considering Moon's eccentricity, triangular libration points remain stable in the Earth-Moon system. Nevertheless, periodic orbits around equilibrium points no longer exist and are replaced by quasi-periodic orbits. Numerical methods are used to study the dynamics around equilibrium points; However, analytical solutions can provide deep insights for understanding the motion around equilibrium points. Lei and $\mathrm{Xu}$ (2014) construct high-order solutions of bounded orbits around $L_{4}$ and $L_{5}$ in the ERTBP, expressed as formal series of four amplitudes: the orbital eccentricity, the long, short and vertical periodic amplitudes. The series expansion up to arbitrary order are constructed by means of Lindsteadt-Poincaré method. Bounded orbits around these points are considered for applications to space missions.

The second scenario analyzed here is the Bicircular Four Body Problem (BCFBP). This dynamical model is a simplified version of the Restricted Four Body Problem. In this case, we suppose that the Earth and the Moon are rotating in circular orbits around their center of mass, and the Earth-Moon barycenter turns in a circular orbit around the center of masses of the Sun-Earth-Moon system (Simó et al., 1995). Although the model is not coherent with the motion of these three bodies due to the assumptions made above, it captures in some sense the basic dynamics of a real four body problem. The equations of motion of the BCFBP in the synodic system can be written in an autonomous fashion; however, $L_{4}$ and $L_{5}$ are no longer equilibrium points, only retaining their geometrical meaning. Using different approaches, Kolenkiewicz and Carpenter (1967), Kolenkiewicz and Carpenter (1968), and Gómez et al. (2001b) have obtained three periodic orbits in the synodical coordinate frame, that have the same period as the Sun. Two of them are linearly stable, lying far away from the triangular libration points of the CRTBP, while the other one, small and slightly unstable. Similarly, two more periodic orbits (mildly unstable) were found, both having period three times of the Sun.

The goal of this work is to investigate the existence of stable regions for small formation flight dynamics (maximum distance between the satellites no greater than few kilometers at most) near bounded solutions around the equilateral equilibrium points of the CRTBP adopting the Elliptic Restricted Three Body Problem (ERTBP) and the BiCircular Four Body Problem (BCFBP) scenarios. Compared to CRTBP, Moon's eccentricity and gravitational perturbation from Sun could approximate the dynamics near triangular libration points more accurately. Similarly, the method based on the linearization of the relative equations of motion with respect to tra- 
jectories around $L_{4}$ is carried out. Additionally, the cost estimate to maintain a formation in these regions or keeping a rigid configuration is computed according to the residual acceleration concept.

The remainder of this paper is organized as follows. Sections 2 and 3 describe the equations of motion of the ERTBP and BCFBP, respectively, and quasi-periodic and periodic solutions about $L_{4}$. Section 4 defines the regions of Zero Relative Radial Acceleration component with respect to a nominal trajectory, as well as, the function to estimate the cost to maintain a formation. Sections 5 and 6 determine the surfaces of zero drift for a family of bounded solutions about $L_{4}$ in the elliptic and bicircular scenarios, respectively, and compute the cost estimate to keep the geometrical configuration of the spacecraft placed in those regions. At last, the conclusions together with the discussion are drawn in Section 7 .

\section{Quasi-periodic orbits around triangular libration points in the ERTBP}

\subsection{The Equations of the ERTBP}

Let us consider two primaries $P_{1}$ of mass $1-\mu$ and $P_{2}$ of mass $\mu$, moving around their barycenter in an elliptic orbit, such that, the total mass of the primaries, their instantaneous distance between them and instantaneous angular velocity are all taken as unity. In addition, the Newton's gravitational constant and the period of the secondary are 1.0 and $2 \pi$, respectively. The differential equations of motion

for a spacecraft $P_{3}$ using a rotating Cartesian rectangular coordinate system with the primaries in the non-dimensional variables are governed by (Szebehely, 1967)

$$
\begin{aligned}
x^{\prime \prime}-2 y^{\prime} & =\frac{1}{1+e \cos f} \frac{\partial \Omega}{\partial x}, \\
y^{\prime \prime}+2 x^{\prime} & =\frac{1}{1+e \cos f} \frac{\partial \Omega}{\partial y}, \\
z^{\prime \prime}+z^{\prime} & =\frac{1}{1+e \cos f} \frac{\partial \Omega}{\partial z},
\end{aligned}
$$

where $e$ is the orbital eccentricity of the secondary, $f$ is the true anomaly of the secondary and $\Omega$ is the pseudo potential function of the three-body problem given by

$$
\Omega=\frac{1}{2}\left(x^{2}+y^{2}+z^{2}\right)+\frac{1-\mu}{r_{1}}+\frac{\mu}{r_{2}}+\frac{1}{2} \mu(1-\mu),
$$


with

$$
\begin{aligned}
& r_{1}=\sqrt{(x+\mu)^{2}+y^{2}+z^{2}}, \\
& r_{2}=\sqrt{(x-1+\mu)^{2}+y^{2}+z^{2}} .
\end{aligned}
$$

The system of differential equations (1) is formulated in the barycentric pulsating synodic coordinate system where the positions of $P_{1}$ and $P_{2}$ in this frame remain fixed at $(-\mu, 0,0)$ and $(1-\mu, 0,0)$, respectively, and $f$ is taken as the time-independent variable. Thus, the first and second derivatives of the coordinates are taken with respect to $f$, i.e., $x^{\prime}=d x / d f, x^{\prime \prime}=d^{2} x / d f^{2}$ (similar expressions for $y$ and $z$ components).

Similarly to the CRTBP scenario, the equations of motion in the ERTBP have five equilibrium points, three of them, denoted by $L_{1}, L_{2}$ and $L_{3}$, aligned with the $x$-axis, are called collinear libration points, and the remaining two points, denoted by $L_{4}$ and $L_{5}$, constituting equilateral triangles with the primaries, are called triangular libration points.

\subsection{Solutions around equilateral equilibrium points in the ERTBP}

In the Earth-Moon Elliptic restricted three body problem, the mass parameter of the system is $\mu=1.215058560962404 \times 10^{-2}$, while the orbital eccentricity of the Moon is $e=0.05488$ (Fitzpatrick, 2012). Because of the perturbation of the Moon's eccentricity, the periodic orbits around triangular libration points no longer exists, and are replaced by quasi-periodic orbits. Lei and Xu (2014) expanded analytical solutions around triangular libration points as formal series of the orbital eccentricity $e$, amplitude of the long periodic motion $\alpha$, amplitude of the short periodic motion $\beta$ and amplitude of vertical periodic motion $\gamma$. In the process of construction, four orders are defined: $N_{1}$ denotes the order of the orbital eccentricity, $N_{2}$ denotes the order of long periodic amplitude, $N_{3}$ denotes the order of short periodic amplitude, and $N_{4}$ denotes the order of vertical amplitude. The total order of the analytical solution is $N=N_{1}+N_{2}+N_{3}+N_{4}$. Thus, the general terms of the formal series for each coordinate are $x_{p i j k}^{l m n r} e^{p} \alpha^{i} \beta^{j} \gamma^{k}, y_{p i j k}^{l m n r} e^{p} \alpha^{i} \beta^{j} \gamma^{k}, z_{p i j k}^{l m n r} e^{p} \alpha^{i} \beta^{j} \gamma^{k}$, where $0 \leq p \leq$ $N_{1}, 0 \leq i \leq N_{2}, 0 \leq j \leq N_{3}, 0 \leq k \leq N_{4}$, with $|l| \leq p,|m| \leq i,|n| \leq j,|r| \leq k$, and $l, m, n, r \in \mathbb{Z}$. The unknown coefficients $x_{\text {pijk }}^{l m n r}, y_{\text {pijk }}^{l m n r}, z_{\text {pijk }}^{l m n r}$ associated with an analytical solution constructed up to order $\left(N_{1}, N_{2}, N_{3}, N_{4}\right)$ are determined from loworder solutions by Lindstedt-Poincaré method (Drazin, 1992), that is, the terms up to order $\left(n_{1}, n_{2}, n_{3}, n_{4}\right), n_{i} \leq N_{i}(i=1, \ldots, 4)$ are to be computed. The analytical 
expressions permit to compute the first and second derivatives of initial states with respect to true anomaly $f$ corresponding to quasi-periodic motions.

In order to check the behavior of a formation in a long and short quasi-periodic orbits near triangular libration points in the Earth-Moon ERTBP, two families of five spatial quasi-periodic orbits around $L_{4}$ during 226 units of dimensionless time (about 1,000 days) are considered, and their projections into the $(x, y)$ and $(x, z)$ planes are presented in Fig. 1 for $\alpha=0.01,0.02,0.03,0.04,0.05$ adim, $\beta=0$, $\gamma=0.03$ adim (Fig. 1a and Fig. 1b), and $\alpha=0, \beta=0.01,0.02,0.03,0.04$, 0.05 adim, $\gamma=0.03$ adim (Fig. 1c and Fig. 1d). Each orbit was obtained by series expansions, taking the construction of analytical solutions truncated at order $(3,3,3,3)$. Three dimensional motion is considered in this section, therefore, no planar formations are carried out. As so, all satellites in the cluster have out-ofplane component. Additionally, analytical solutions truncated at order $(3,3,3,3)$ for $e=0.05488, \alpha<0.05$ adim, $\beta<0.05$ adim, $\gamma<0.03$ adim present a domain of convergence about $1.0 \times 10^{-5}$ adim, more than enough for practical purposes $(1$ adim is about $384,405 \mathrm{~km}$ ). The system of differential equations (1) were integrated numerically considering the initial states given by the analytical expressions and using the Bulirsch-Stoer algorithm (Stoer and Bulirsch, 1980; Press et al., 1992), programmed in the $\mathrm{C}$ language, choosing a dimensionless step size $h=1.0 \times 10^{-3}$ and setting a tolerance of $1.0 \times 10^{-9}$.
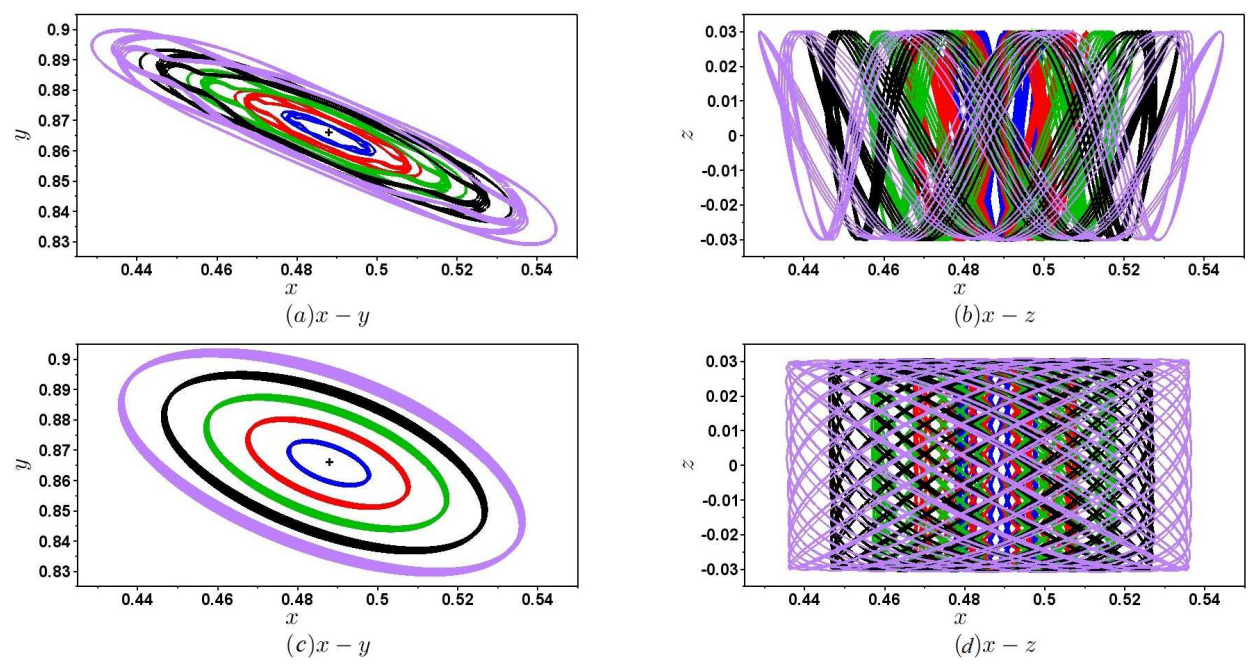

Figure 1: (a)-(d) Projections into the $(x, y)$ and $(x, z)$ planes of a long and short quasi-periodic family around $L_{4}$ in the Earth-Moon ERTBP for $\alpha$ and $\beta$ equal to 0.01, 0.02, 0.03, 0.04, 0.05 adim, and $\gamma=0.03$ adim. 


\section{Periodic orbits around triangular libration points in the BCFBP}

\subsection{The Equations of the BCFBP}

Taking into account the Sun's gravitational force in the Earth-Moon system, the BCFBP is formulated under the following assumptions: (i) the movements of the Sun, Earth and Moon take place in the same plane; (ii) the Earth and Moon move around their barycenter in a circular orbit; (iii) the Sun and Earth-Moon barycenter move around their common center in circular orbits. This dynamical model is a modified version of the Earth-Moon CRTBP and catches the basic dynamics of the real four-body problem.

Similarly, let $\mu$ be the mass of the Moon, $1-\mu$ the mass of the Earth and $\mu_{S}$ the mass of the Sun. Taking as unity the distance between the Earth and the Moon, let $a_{S}$ the distance from the Earth-Moon barycenter to the Sun. The equations of motion in the Earth-Moon synodic system, centered at the Earth-Moon barycenter are given by (Gómez et al., 2001b)

$$
\begin{aligned}
\ddot{x}-2 \dot{y} & =\frac{\partial \Omega^{S}}{\partial x}, \\
\ddot{y}+2 \dot{x} & =\frac{\partial \Omega^{S}}{\partial y}, \\
\ddot{z}+\dot{z} & =\frac{\partial \Omega^{S}}{\partial z}, \\
\dot{\theta_{S}} & =\omega_{S},
\end{aligned}
$$

where $\omega_{S}$ is the angular speed of the Sun in the synodical system, $\theta_{S}$ is the phase angle of the Sun relative to the Earth-Moon line, and $\Omega^{S}$ is the perturbed pseudo potential caused by the Sun,

$$
\begin{aligned}
\Omega^{S}= & \frac{1}{2}\left(x^{2}+y^{2}+z^{2}\right)+\frac{1-\mu}{r_{1}}+\frac{\mu}{r_{2}}+\frac{1}{2} \mu(1-\mu) \\
& +\mu_{S}\left(\frac{1}{r_{S}}-\frac{1}{a_{S}^{2}}\left(x \cos \theta_{S}-y \sin \theta_{S}\right)\right),
\end{aligned}
$$

with

$$
r_{S}=\sqrt{\left(x-a_{S} \cos \theta_{S}\right)^{2}+\left(y+a_{S} \sin \theta_{S}\right)^{2}+z^{2}}
$$

The positions of the Earth, the Moon, and the Sun in the synodical system are $(-\mu, 0,0),(1-\mu, 0,0),\left(a_{S} \cos \theta_{S},-a_{S} \sin \theta_{S}, 0\right)$, respectively. Additionally, even

though the system of differential equations (4) is autonomous, $L_{4}$ and $L_{5}$ are no longer equilibrium points, keeping only a geometrical meaning. 


\subsection{Solutions around triangular libration points in the BCFBP}

In the Sun-Earth-Moon Bicircular four body problem, we take the mass parameter $\mu=1.215058560962404 \times 10^{-2}$, the mass of the Sun $\mu_{S}=328900.55$, the distance between the Sun and the Earth-Moon barycenter $a_{S}=388.8111430233514$ adim, and the angular speed of the Sun $\omega_{S}=0.92519598551828964$. These values are consistent with the ones used by Gómez et al. (2001b). Fig. 2 shows a set of trajectories, obtained by initial conditions of five planar periodic orbits of the EarthMoon CRBTP around $L_{4}$, with amplitude of long periodic motion $\alpha=0.01,0.02$, 0.03, 0.04, 0.05 adim, and perturbed by the Sun using the BCFBP with initial phase angle $\theta_{S}=0$ at 226 units of dimensionless time (about 1,000 days), i.e., the initial angle between the Sun-Earth and Earth-Moon lines is equal to zero. As can be seen in Fig. 2, periodic orbits in the vicinity of triangular points in the Earth-Moon CRTBP become quasi-periodic orbits when Sun's gravitational attraction is included, that expand up to 0.6 adim approximately (about $230,000 \mathrm{~km}$ ) and contract to some 0.02 adim (about $7,600 \mathrm{~km}$ ) from $L_{4}$, and for $\alpha>0.03$ adim, the spacecraft left the libration-point-centered motion. This fact was already reported by Feldt and Schulman (1966), Tapley and Schultz (1965), and Tapley and Schultz (1968), showing the lack of stability of the motion close to $L_{4}$ and $L_{5}$; unlike the perturbation from Moon's eccentricity in the Earth-Moon ERTBP that keeps up the stability of the triangular libration points as shown in Fig. 1. Therefore, the objective of this investigation is to choose a set of well-behaved bounded solutions about $L_{4}$ to place a formation when solar gravitational force is considered.

The effects of the initial configuration of the Sun-Earth-Moon were studied by Tapley and Lewallen (1964), Wolaver (1965), and more recently, by Munoz (2008). The problem is to determine the initial phase angle $\theta_{S}$, i.e., the initial position of the Sun, that minimizes the maximum displacement from $L_{4}$. Wolaver (1965), using the approximation of the BCFBP and linearizing the equations of motion in the vicinity of $L_{4}$, obtained three initial positions of the Sun that showed a maximum displacement of $5,349 \mathrm{~km}$ from $L_{4}$ for 400 days. However, this solution does not persist in a more realistic ephemeris-based model. On the other hand, Tapley and Lewallen (1964), including also the inclination of the Sun's orbit in the Sun-EarthMoon system, found that the motion of the spacecraft initially at $L_{4}$ is very dependent on the initial position of the Sun and that the amplitude of motion about $L_{4}$ and $L_{5}$ is greater than what was predicted by the linearized equations. Similarly, Munoz (2008) obtained an initial value of $\theta_{S}$ in the Sun-Earth-Moon BCFBP, so that, a spacecraft initially at rest at $L_{4}$ remained within $30,000 \mathrm{~km}$ of $L_{4}$ for a propagation time of 7,000 days. However, the resulting trajectory was extremely sensitive to the initial value of $\theta_{S}$. Therefore, using more realistic model of the Sun-Earth-Moon 

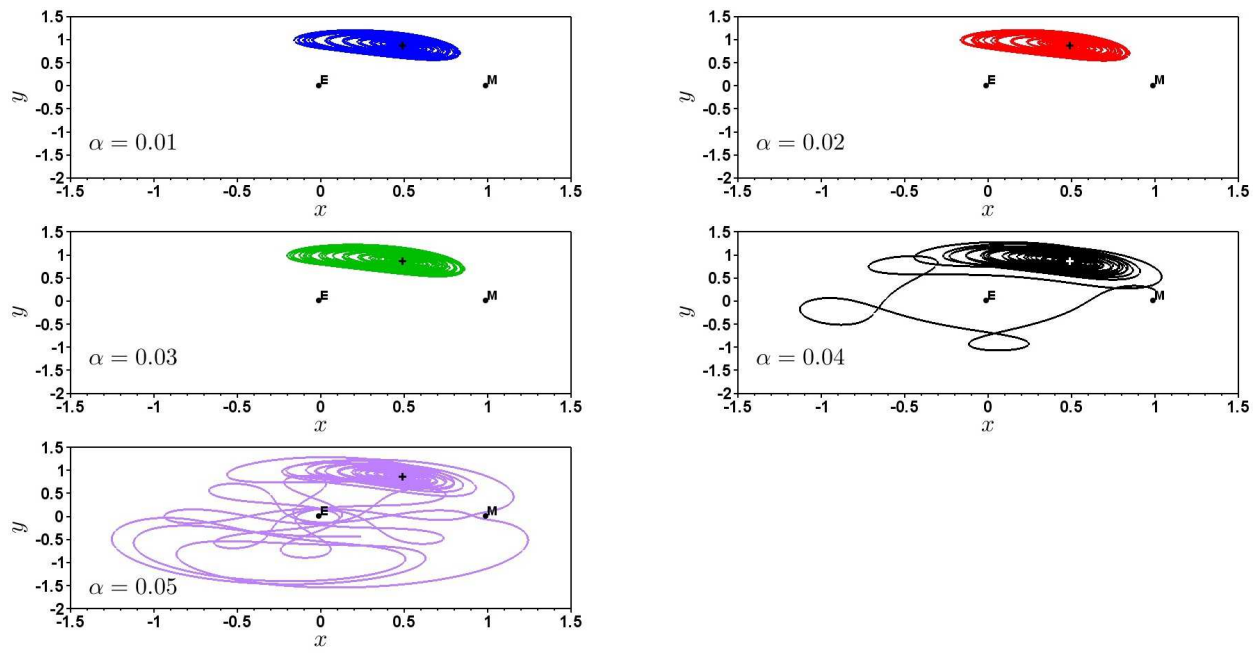

Figure 2: Quasi-periodic orbits around $L_{4}$ in the Sun-Earth-Moon BCFBP obtained by periodic orbits near $L_{4}$ in the Earth-Moon CRTBP for $\alpha=0.01,0.02,0.03,0.04,0.05$ adim, initial phase angle $\theta_{S}=0$, and numerically integrated at 226 units of dimensionless time (about 1,000 days).

system, Munoz (2008) studied the motion of a spacecraft near the triangular libration points in the Earth-Moon system using the SPICE ephemerides, and found in the year 2007, 12 and 13 epochs in the $L_{4}$ and $L_{5}$ cases, respectively, such that the spacecraft would not exhibit the expansion and contraction as shown in Fig. 2, and remain in a close vicinity about triangular points for at least 3,000 days. But in this model again, the resultant motion was sensitive to the initial configuration.

On the other hand, despite the wide departures from $L_{4}$ described above, Schechter (1968) showed the existence of two periodic orbits around $L_{4}$ whose period is the same as the period of the Sun's perturbation: One stable, with a semi-major axis of about $97,000 \mathrm{~km}$, and another unstable, and slightly larger than the stable one. Schechter (1968) also concluded that the triangular points are no longer stable. Similarly, Kolenkiewicz and Carpenter (1967), using the Huang's model (Huang, 1960) for the Sun-Earth-Moon system, found two stable, periodic orbits, larger than what Schechter (1968) predicted $(145,000 \mathrm{~km})$, and one small, unstable orbit very close to $L_{4}$, in agreement with Schechter (1968)'s conclusion. Later, Gómez et al. (2001b), using a continuation method to pass from the Earth-Moon CRTBP to the Sun-EarthMoon BCFBP, found three periodic orbits with initial phase angle $\theta_{S}=0$, whose period is equal to the period of revolution of the Sun in the Earth-Moon synodical system, i.e., 6.791 units of dimensionless time (about 29 days). Those orbits, denoted by $A, B$, and $C$, are shown in Fig. 3 and Fig. 4 in the Earth-Moon rotating frame 
centered in the Earth-Moon barycenter. Although orbits $A$ and $B$ are quite similar, the initial condition, marked with a small circle on the orbit, is different. The periodic orbits $A$ and $B$ are stable solutions about $L_{4}$, i.e., the norm of the eigenvalues of the monodromy matrix associated with the two trajectories are less than one. Those orbits are very large, about 0.6 adim $(230,000 \mathrm{~km})$ along the $x$-axis and 0.4 adim $(153,000 \mathrm{~km})$ along the $y$-axis. Orbit $C$ is unstable periodic orbit about $L_{4}$. This orbit remains within 0.02 adim $(7,600 \mathrm{~km})$ of the libration point.

Finally, Gómez et al. (2001b) found two larger, unstable periodic orbits with initial phase angle $\theta_{S}=0$, whose period is three times larger than the solar perturbation, i.e., 20.373 units of dimensionless time (about 88 days). Those orbits, denoted by $D$ and $E$, are shown in Fig. 5 . In the same way, orbits $D$ and $D$ are quite similar, but the initial condition, marked with a small circle on the orbit, is different. When a more realistic model is considered, there remain quasi-periodic orbits around $L_{4}$ and $L_{5}$ as shown by Díez et al. (1991), Jorba (2000) and Gómez et al. (2001c). The five planar periodic orbits about $L_{4}$ described in this section will be chosen and analyzed in this study as nominal trajectories, such that, a small planar formation is flying near them.
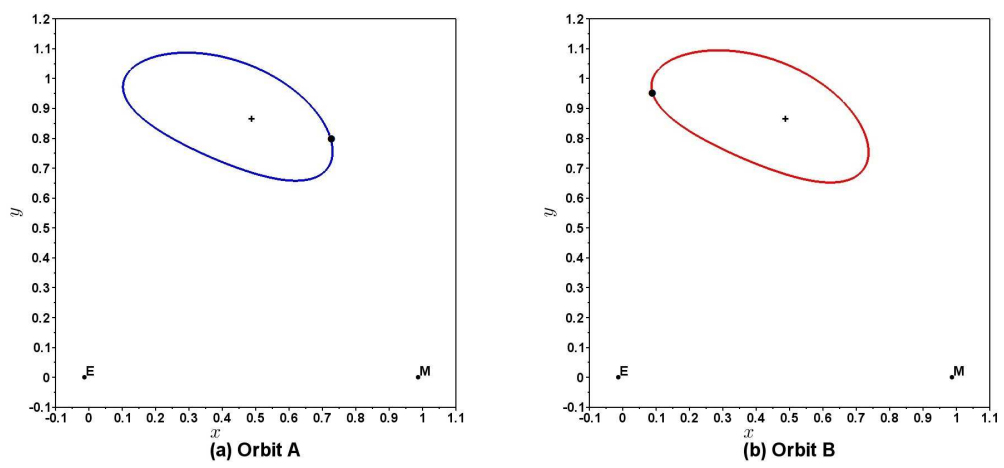

Figure 3: (a)-(b) Stable periodic orbits about $L_{4}$ in the Sun-Earth-Moon BCFBP with initial phase angle $\theta_{S}=0$ and period equal to the period of revolution of the Sun in the Earth-Moon synodical system. The initial condition is marked with a small circle

\section{Natural regions suitable for small formation}

\subsection{Equations of local dynamics of SFF derived via variational equations}

For the purpose of this research, the satellite located at the center of the relative frame, will be called 'chief' satellite and the satellite operating in the vicinity of the chief will be called 'deputy' (see Fig. 6). In order to avoid expansion or contraction 

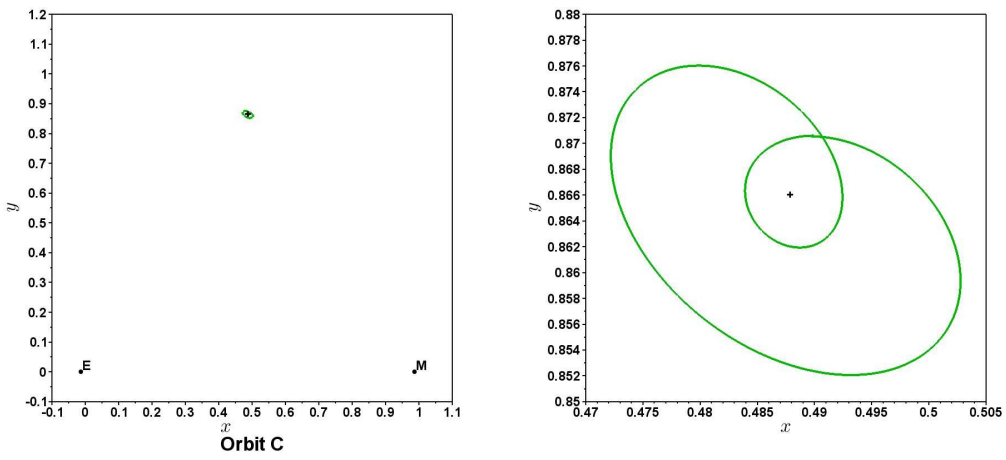

Figure 4: Unstable periodic orbit about $L_{4}$ in the Sun-Earth-Moon BCFBP with initial phase angle $\theta_{S}=0$ and period equal to the period of revolution of the Sun in the Earth-Moon synodical system. The right figure presents a magnification of the orbit C.
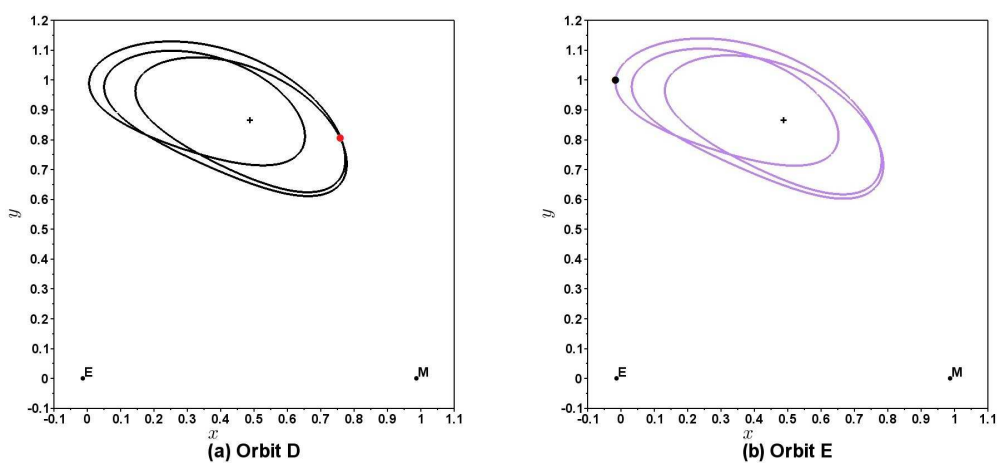

Figure 5: (a)-(b) Unstable periodic orbits about $L_{4}$ in the Sun-Earth-Moon BCFBP with initial phase angle $\theta_{S}=0$ and period three times of the revolution of the Sun in the Earth-Moon synodical system. The initial condition is marked with a small circle.

with respect to the chief satellite, the existence of regions with zero relative velocity and zero relative radial acceleration (ZRRA) may be exploited (Perea, 2006). Assuming that the radius of the formation geometry structure (largest separation between spacecraft) is small, no greater than few kilometers at most, then a linear approach gives all the relevant information about the local dynamics of the problem.

Let

$$
X=(x, y, z, \dot{x}, \dot{y}, \dot{z})^{T}
$$

be the vector that describes the position and velocity of the deputy satellite in the rotating frame, where superscript " $T$ " means transpose. Given a reference solution $X_{h}$ around $L_{4}$, i.e, the corresponding trajectory that is supposedly followed by the 
chief satellite, then the linear variational equations of motion about the reference solution in the matrix form is given by

$$
\delta \dot{X}(t)=A(t) \delta X(t)
$$

where $\delta X(t)=X_{h}(t)-X(t)$ represents the variations of the deputy satellite with respect to the chief's path. The coordinates of $\delta X(t)$ are defined in a coordinate system $(\hat{x}, \hat{y}, \hat{z})$ parallel to the rotating coordinate system $x, y, z$ and centered at $X_{h}(t)$ as shown in Fig. 6. The matrix $A(t)$ is time-varying of the form

$$
A(t)=\left(\begin{array}{cc}
0_{3 \times 3} & I_{3 \times 3} \\
F & J
\end{array}\right)
$$

where the matrices $F$ and $J$ are defined as

$$
\begin{aligned}
F & =\left(\begin{array}{ccc}
\Omega_{x x}^{S} & \Omega_{x y}^{S} & \Omega_{x z}^{S} \\
\Omega_{y x}^{S} & \Omega_{y y}^{S} & \Omega_{y z}^{S} \\
\Omega_{z x}^{S} & \Omega_{z y}^{S} & \Omega_{z z}^{S}
\end{array}\right), \\
J & =\left(\begin{array}{ccc}
0 & 2 & 0 \\
-2 & 0 & 0 \\
0 & 0 & 0
\end{array}\right) .
\end{aligned}
$$

The symbol $\Omega_{i j}^{S}$ stands for the partial derivatives $\partial^{2} \Omega^{S} / \partial i \partial j$ for $i, j=x, y, z$, and these partials are evaluated along the reference trajectory. In the ERTBP scenario, the time derivative in (8) is taken with respect to true anomaly $f$ and the matrix $F$ is of the form

$$
F=\frac{1}{1+e \cos f}\left(\begin{array}{ccc}
\Omega_{x x} & \Omega_{x y} & \Omega_{x z} \\
\Omega_{y x} & \Omega_{y y} & \Omega_{y z} \\
\Omega_{z x} & \Omega_{z y} & \Omega_{z z}
\end{array}\right) .
$$

\subsection{Zero Relative Radial Accelerations Cones}

Writing the variations of the deputy satellite as $\delta X=(\delta r \delta \dot{r})^{T}$, where $\delta r=$ $(\delta x, \delta y, \delta z)^{T}$, the linear system $(8)$ becomes

$$
\left(\begin{array}{c}
\delta \dot{r} \\
\delta \ddot{r}
\end{array}\right)=\left(\begin{array}{cc}
0_{3 \times 3} & I_{3 \times 3} \\
F & J
\end{array}\right)\left(\begin{array}{c}
\delta r \\
\delta \dot{r}
\end{array}\right) .
$$

Assuming a small distance between the deputy and chief satellites, and relative velocity in an ideal direction equal to zero, from Eq.(13), the relative acceleration is then

$$
\delta \ddot{r}=F \delta r .
$$




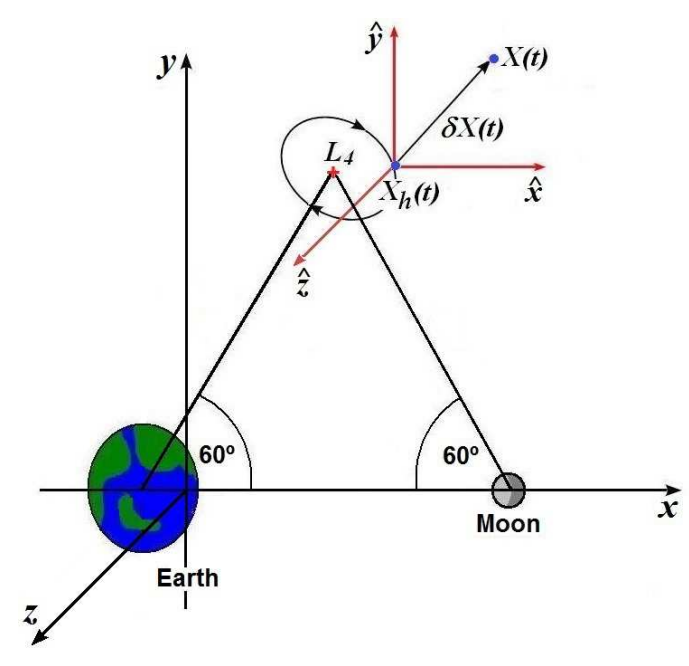

Figure 6: Illustration of a satellite formation flying about $L_{4} . X_{h}(t)$ and $X(t)$ denote the corresponding trajectories of the chief and deputy satellites, respectively, $\delta X(t)$ represents the variations of the deputy satellite with respect to chief's path in a coordinate system parallel to the rotating coordinate system $x, y, z$ and centered at $X_{h}(t)$ (not to scale).

Therefore, the locations such that radial component of the relative acceleration is zero satisfies the equation

$$
\delta r^{T} F \delta r=0 .
$$

Eq.(15) represents a quadratic surface, which depends on the point $X_{h}(t)$ selected along the reference solution of (1) or (4).

Regions with Zero Relative Radial Acceleration (ZRRA) component can also be computed numerically. Given a certain reference solution, let us consider a point along the trajectory that represents the state of the chief spacecraft. Now, centered at the location of the chief satellite, let us define a sphere of radius equal to $2 \mathrm{~km}$ in the configuration space, such that, the velocity of all the points on the sphere are assumed to be equal to zero (zero relative velocity condition). Using polar coordinates, the locations of the deputy vehicle on the sphere can be parametrized by the angles $\psi$ and $\phi$ as illustrated in Fig. 7. The relative acceleration, that corresponds to each of the states of the sphere, can be evaluated using the right side of Eqs. (1) or (4), whose dot product with $\delta r(\psi, \phi)$ will permit to obtain the desired radial component.

\subsection{Cost Estimate to Maintain a Spacecraft in a Formation}

The cost estimate to maintain two or more satellites in formation can be evaluated using the concept of residual acceleration. Thus, given a certain reference 


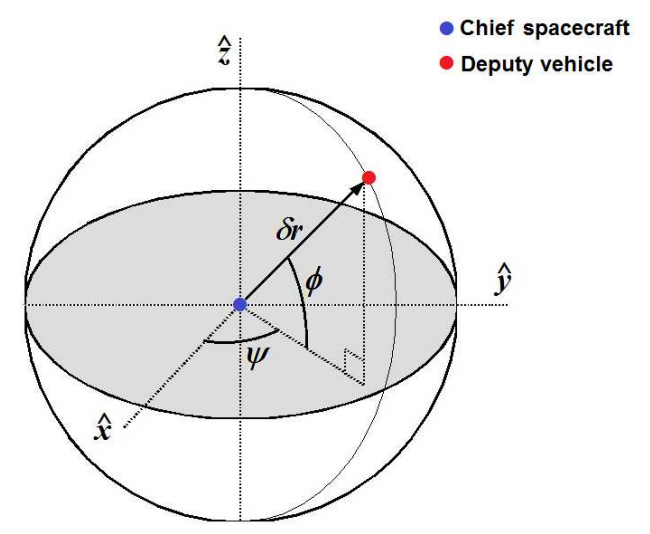

Figure 7: Sphere of radius equal to $2 \mathrm{~km}$ centered at the location of the chief satellite in the configuration space, such that, the velocity of all the points on the sphere are assumed to be equal to zero, and the locations of the deputy vehicle on the sphere are parametrized using spherical coordinates denoted by the angles $\psi$ and $\phi$.

trajectory $X_{h}(t)$ around $L_{4}$, suppose that a spacecraft is following an artificial trajectory around it, i.e., there exists a continuous effort (control) applied on it to maintain this trajectory. Let us denote by $\delta r_{a}(t)$ the relative position at time $t$. If there is no continuous effort applied on the spacecraft, then the function $\delta r_{a}(t)$ will satisfy Eq.(13). However, if a control is applied on the spacecraft, there is a residual acceleration as a consequence of the maneuvers applied on it. Hence, denoting by $R_{a}$ the residual acceleration, it can be computed using Eq.(13):

$$
R_{a}=\delta \ddot{r}_{a}-\left(F \delta r_{a}+J \delta \dot{r}_{a}\right)
$$

Therefore, the cost estimate $\Delta V$ to maintain the spacecraft on an artificial trajectory until a time $T$ is simply:

$$
\Delta V=\int_{0}^{T}\left\|\boldsymbol{R}_{\boldsymbol{a}}(t)\right\| \mathrm{d} t
$$

\section{Zero Relative Acceleration Cones in a family of solutions about $\boldsymbol{L}_{4}$}

\subsection{The Earth-Moon ERTBP scenario}

Since the analytical expression for the ZRRA derived by Eq.(15) represents a quadratic surface, then Eq.(15) is transformed into its canonical form using a change of coordinates. Because of matrix $F$ is symmetric, i.e., $F=F^{T}$, it can be diagonalized and takes the form

$$
F=P \Lambda P^{T},
$$


where $P$ is the orthogonal matrix and $\Lambda$ is the real diagonal matrix, and are of the form

$$
\begin{aligned}
P & =\left(\begin{array}{lll}
V_{1} & V_{2} & V_{3}
\end{array}\right) \\
\Lambda & =\left(\begin{array}{ccc}
\lambda_{1} & 0 & 0 \\
0 & \lambda_{2} & 0 \\
0 & 0 & \lambda_{3}
\end{array}\right) .
\end{aligned}
$$

The real values $\lambda_{1}, \lambda_{2}$, and $\lambda_{3}$ represent the eigenvalues of $F$. The column vectors $V_{1}, V_{2}$, and $V_{3}$ denote the eigenvectors of $F$ and form an orthonormal basis that identifies the principal directions of the quadratic surface.

Now, defining the vector $\delta \eta=\left(\delta \eta_{1} \delta \eta_{2} \delta \eta_{3}\right)^{T}$, such that, $\delta \eta=P^{T} \delta r$, then Eq. (15) can be rewritten in the following form

$$
\lambda_{1} \delta \eta_{1}^{2}+\lambda_{2} \delta \eta_{2}^{2}+\lambda_{3} \delta \eta_{3}^{2}=0
$$

Eq.(21) represents a second order surface and there are 17 standard-form types (Hilbert and Cohn-Vossen, 1999). However, since the right side of Eq.(21) is equal to zero, then there are only two types of quadratic surfaces to be investigated: the elliptic cones and the intersecting planes. To determine the type of quadratic surface out of these two forms in Eq.(21), the following components of matrix $\Lambda$ are examined: i) The determinant $\Delta$ of matrix $\Lambda$, ii) the rank $\rho$ of $\Lambda$, and iii) the sign of the eigenvalues $\lambda_{1}, \lambda_{2}$, and $\lambda_{3}$. Table 1 describes the properties of the two types of quadratic surfaces that are determined in this investigation by examining only the real domain in Eq. (21). Thus, the specific type of quadratic surface depends on the given reference orbit about $L_{4}$ and the corresponding properties of matrix $\Lambda$.

\begin{tabular}{cccc}
\hline Type of surface & Sign of $\Delta$ & $\rho$ & The sign of the nonzero $\lambda_{i}$ are the same \\
\hline Cones & Positive/Negative & 3 & No \\
Planes & Zero & 2 & No \\
\hline
\end{tabular}

Table 1: Properties of the elliptic cones and intersecting planes

Another approximation is derived by Héritier and Howell (2014) by considering the locations along a halo orbit near the $L_{2}$ Sun-Earth libration point, such that, the relative acceleration is less than a small positive coefficient denoted by $\varepsilon$. Small ellipsoids are then derived along the reference path, that maintain the mutual distance between the spacecraft and the pointing direction orienting the formation. In this case, the small ellipsoids correspond to the following quadratic inequality

$$
\delta r^{T} F^{T} F \delta r \leq \varepsilon .
$$


To derive these ellipsoids, various small distances between the chief and deputy spacecraft are examined, while varying the value for the $\varepsilon$ coefficient. The eigenvectors of the matrix $F$ also represent the principal directions corresponding to the ellipsoids and, therefore, the ellipsoids possess the same orientation as the cones derived in this work given the same reference trajectory.

The eigenvalues $\lambda_{1}, \lambda_{2}$, and $\lambda_{3}$ associated to the matrix $F$ in Eq.(18) are then examined considering the family of quasi-periodic orbits defined in Fig. 1. As can be seen in Fig. 8, the sign of the eigenvalues $\lambda_{1}$ and $\lambda_{3}$ is never equal or close to zero along the family of long quasi-periodic orbits. However, examining the behavior of the sign of $\lambda_{2}$, it was found that the eigenvalue $\lambda_{2}=0(\operatorname{rank} \rho=2)$ at some locations along the reference orbit for $\alpha \geq 0.04 \operatorname{adim}(\beta=0, \gamma=0.03 \mathrm{adim})$. Similarly, it was obtained that $\lambda_{2}=0$ at some locations along the reference orbit for $\beta \geq 0.02$ adim $(\alpha=0, \gamma=0.03 \mathrm{adim})$. Hence, fixing the vertical periodic motion, only elliptic cones exist (rank $\rho=3$ ) in the vicinity of a family of long and short quasi-periodic orbits when $\alpha<0.04$ adim and $\beta<0.02$ adim (near enough $L_{4}$ ), respectively. On the other hand, at some positions on the reference quasi-periodic orbits, elliptic cones become intersecting planes ( $\operatorname{rank} \rho=2$ ) when $\alpha \geq 0.04$ adim and $\beta \geq 0.02$ adim (far enough from $L_{4}$ ), changing their orientation.
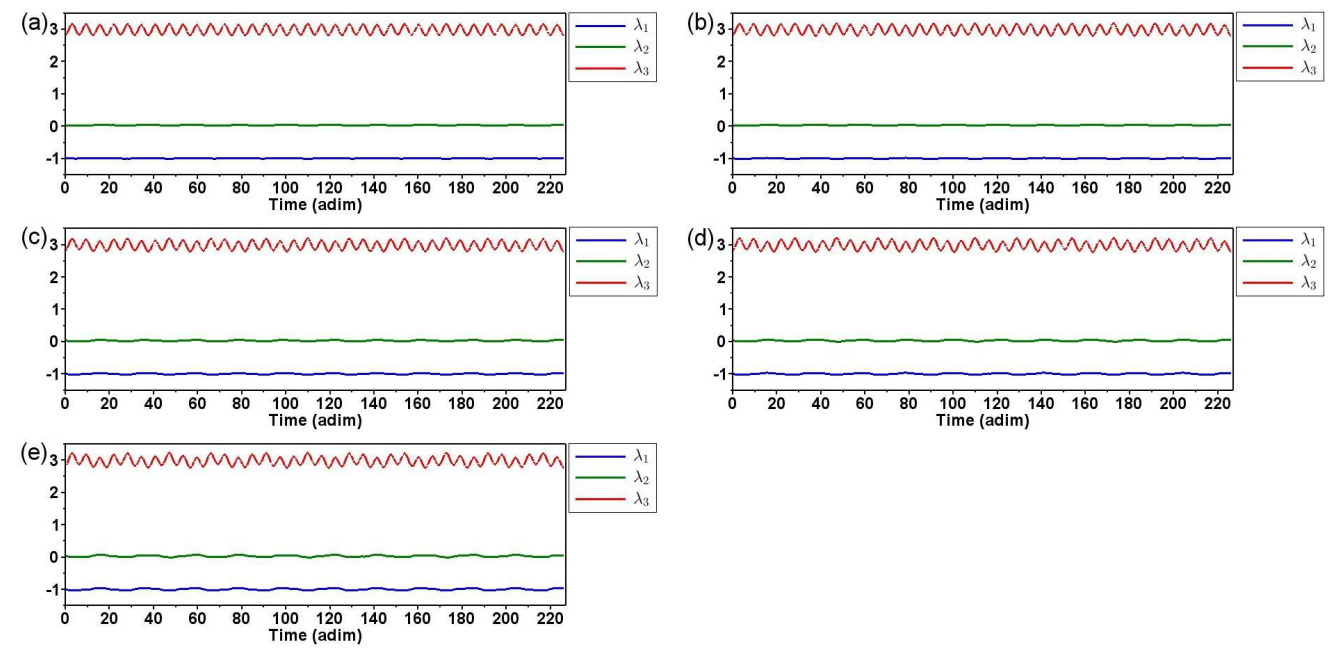

Figure 8: (a)-(e) Eigenvalues $\lambda_{1}, \lambda_{2}$, and $\lambda_{3}$ associated to the matrix $F$, considering the family of long quasi-periodic orbits for $\alpha=0.01,0.02,0.03,0.04,0.05$ adim, and $\gamma=0.03$ adim.

To illustrate how the relative radial acceleration varies as a function of angles $\psi$ and $\phi$ (defined previously in Fig. 7), Fig. 9 shows the dimensionless relative radial acceleration component between the deputy satellite and chief spacecraft, computed 
numerically using the right side of Eq.(1), corresponding to the family of long and short quasi-periodic orbits, respectively, at specific locations such that the eigenvalue $\lambda_{2}$ does not approach to zero. The corresponding sphere of radius $2 \mathrm{~km}$ shows there appear two maxima, associated to the unstable directions, and two minima, related to the stable ones. However, the relative radial acceleration never reaches zero. Therefore, a small positive coefficient $\varepsilon$ may be introduced for the computation of low drift regions, and similarly, Eq.(15) could be rewritten as

$$
\delta r^{T} F \delta r \leq \varepsilon
$$
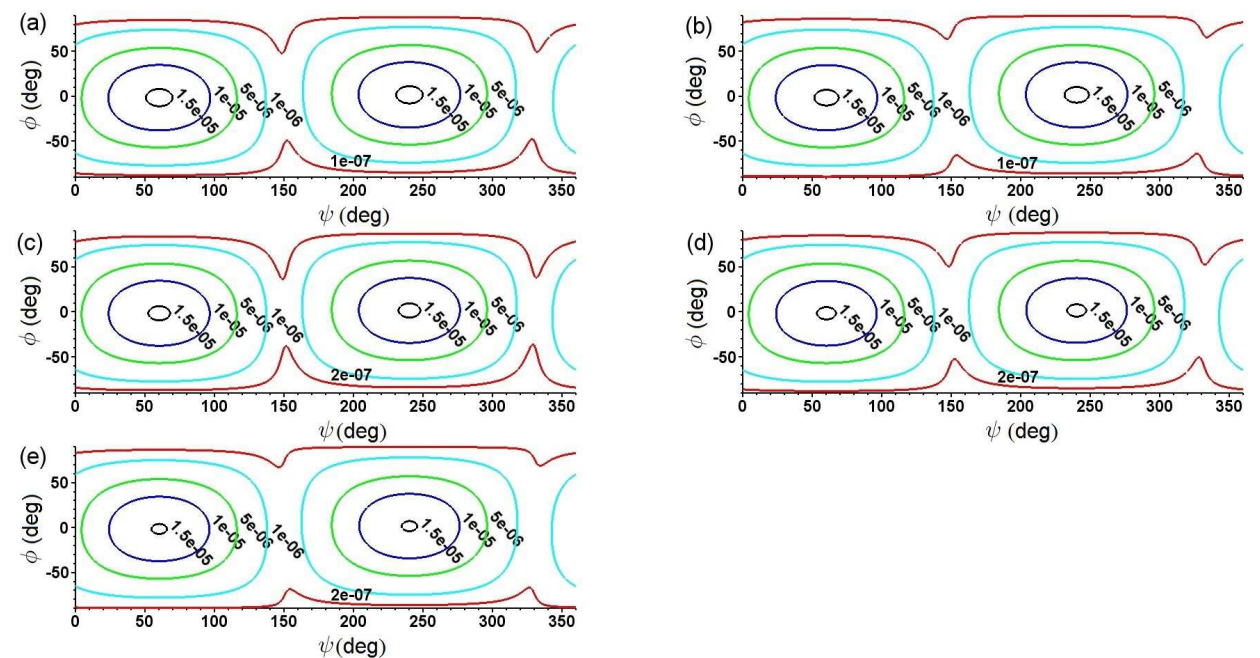

Figure 9: (a)-(e) Dimensionless relative radial acceleration component with respect to angles $\psi$ and $\phi$ at the chief location at time $t=70$ days from the initial location, considering the family of long quasi-periodic orbits.

\subsection{Cost Estimate to maintain a formation on the ZRRA cones}

In this section we estimate the cost to maintain a formation moving within the ZRRA cones, considering a separation along the reference solution between the deputy and chief spacecraft of $2 \mathrm{~km}$ and four different geometrical configurations. This separation is also considered in the Sun-Earth-Moon BCFB scenario. For simplicity, we will assume that the formation has only three spacecraft: two deputy vehicles at the edges of a segment on the $\left(\delta \eta_{1}, \delta \eta_{3}\right)$ - or $\left(\delta \eta_{1}, \delta \eta_{2}\right)$ planes and the chief satellite at the middle point. The third satellite will move along the reference orbits 
shown in Fig. 1 without any control acting on it. The length of the segment will then be $4 \mathrm{~km}$.

Fig. 10 illustrates the four geometrical configurations with respect to the orthonormal space generated by the eigenvectors $V_{1}, V_{2}, V_{3}$ defined previously. As the central spacecraft moves along the reference orbit, the first and second formations, considered in Fig. 10, set the two deputy vehicles on the $\left(\delta \eta_{1}, \delta \eta_{3}\right)$-plane, forced to be always on a segment parallel to the initial one, keeping their mutual distances fixed. Similarly, the third and fourth formations set the two deputy vehicles on the $\left(\delta \eta_{1}, \delta \eta_{2}\right)$-plane. If the eigenvalue $\lambda_{2}$ does not approach to zero along a reference path, the four configurations will be considered, otherwise only the first and second ones will be considered to avoid the singularity that appears as a result of the inclination of the intersecting planes. Additionally, segments on $\left(\delta \eta_{2}, \delta \eta_{3}\right)$-plane will not be defined since the elliptic cones do not intersect this plane when $\lambda_{2}>0$.

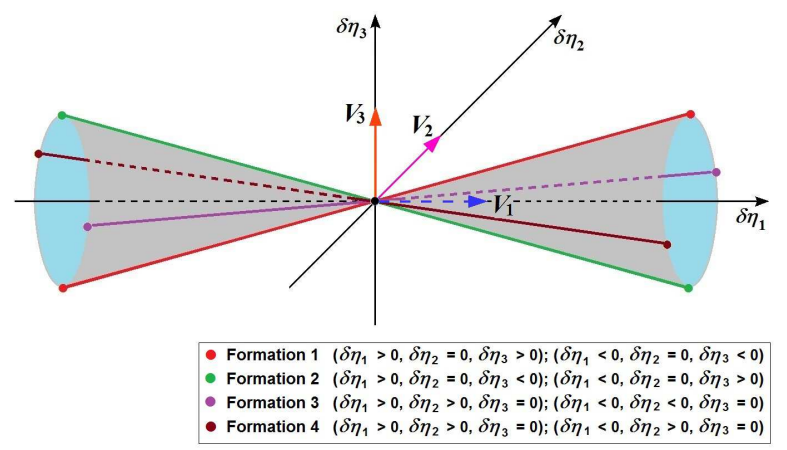

Figure 10: Four geometrical configurations with respect to the orthonormal space generated by the eigenvectors $V_{1}, V_{2}, V_{3}$ of matrix $F$. The chief spacecraft is placed at the middle of a segment defined by the two deputy satellites. The first and second formations set the two deputy vehicles at the edges of the segment defined on the $\left(\delta \eta_{1}, \delta \eta_{3}\right)$-plane, forced to be always on a segment parallel to the initial one, keeping their mutual distances fixed. Similarly, the third and fourth formations set the two deputy vehicles on the $\left(\delta \eta_{1}, \delta \eta_{2}\right)$-plane.

The relative position vector $\delta r_{a}$, from the initial location along the reference orbit to the deputy satellite trajectory during 226 units of dimensionless time (about 1, 000 days), will be computed solving Eq.(21) and using the fact that $\delta r_{a}=P \delta \eta$, where $P$ is the orthogonal matrix defined previously in Eq.(19). The first and second derivatives of the coordinates of vector $\delta r_{a}$ with respect to time will be approximated by a central finite difference of eighth-order accuracy (Fornberg, 1988). Finally, the coordinates of residual acceleration vector $R_{a}$ will be computed using Eq.(16) and, the cost estimate $\Delta V$ to maintain the formation within the ZRRA cones will be determined numerically integrating the expression (17) with $T=226$ by the Trapezoid method. 
Hence, let us denote by $\Delta V_{i}$, where $i=1,2,3,4,5$, the cost estimate for the quasi-periodic orbit with $\alpha=0.01,0.02,0.03,0.04,0.05$, respectively. Table 2 shows the $\Delta V_{i}(\mathrm{~m} / \mathrm{s})$ for the four geometrical configurations along the family of long quasi-periodic orbits shown in Fig. 1. Similar values were obtained for the family of short quasi-periodic orbits. As can be seen in Table 2, for the first and second formations the cost estimate practically remains constant. The same situation presents the last two formations. On the other hand, the costs estimated in Table 2 show that, although the Moon's eccentricity affects the periodic behavior of long and short period orbits found in the Earth-Moon CRTBP, the formation keeping $\Delta V$ requirements are very small for all four configurations. This fact highlights the small variations in the mutual distance between the spacecraft when the formation would be aligned in the direction of the zero drift regions. For instance, placing a planar formation at the low drift regions along a family of periodic orbits near and far from $L_{4}$ in the Earth-Moon CRTBP scenario, Salazar et al. (2014) estimated, using the residual acceleration concept, an average required change in velocity of 1 $\mathrm{cm} / \mathrm{s} /$ year to control the formation; whereas Wong (2009) computed a higher cost, an average of $10 \mathrm{~cm} / \mathrm{s} /$ year, using Linear Quadratic Regulator (LQR) control for reference in-plane elliptical relative trajectories near the Earth-Moon $L_{4}$ point in the same scenario.

\begin{tabular}{lcccccc}
\hline & $\begin{array}{c}\alpha \\
(\operatorname{adim})\end{array}$ & $\begin{array}{c}\Delta V_{1} \\
(\mathrm{~m} / \mathrm{s})\end{array}$ & $\begin{array}{c}\Delta V_{2} \\
(\mathrm{~m} / \mathrm{s})\end{array}$ & $\begin{array}{c}\Delta V_{3} \\
(\mathrm{~m} / \mathrm{s})\end{array}$ & $\begin{array}{c}\Delta V_{4} \\
(\mathrm{~m} / \mathrm{s})\end{array}$ & $\begin{array}{c}\Delta V_{5} \\
(\mathrm{~m} / \mathrm{s})\end{array}$ \\
\hline Formation 1 & 0.01 & 4.1789 & 4.1793 & 4.1798 & 4.1804 & 4.1810 \\
Formation 2 & 0.02 & 4.1747 & 4.1752 & 4.1757 & 4.1763 & 4.1769 \\
Formation 3 & 0.03 & 0.4493 & 0.4448 & 0.4367 & & \\
Formation 4 & 0.04 & 0.4495 & 0.4450 & 0.4369 & & \\
\hline
\end{tabular}

Table 2: Cost estimate $\Delta V_{i}(\mathrm{~m} / \mathrm{s})$ to maintain two deputy spacecraft within the ZRRA cones defined along the family of long quasi-periodic orbits shown in Fig. 1, during 226 units of dimensionless time (about 1,000 days), and keeping a fixed distance of $2 \mathrm{~km}$ between the deputy and chief spacecraft.

Finally, analyzing the cost estimate, we note that the $\Delta V$ requirements are less when the spacecraft are set on the plane generated by eigenvectors $V_{1}$ and $V_{2}$ (see Fig. 10). Since the coordinates of each formation were defined in the orthonormal space $\left\{V_{1}, V_{2}, V_{3}\right\}$, an easier way to see the deputy vehicle's orientation followed along the reference orbits in each formation, is to determine its spherical coordinates with respect to deputy spacecraft, defined in Fig. 7. For simplicity, only the orientation for the long quasi-periodic orbits will be determined.

Similarly, let us denote by orbit $1,2,3,4$, and 5 , the quasi-periodic orbits shown 
in Fig. 1, such that, $\alpha=0.01,0.02,0.03,0.04,0.05$ adim, i.e, orbit 1 corresponds to the smallest quasi-periodic orbit and orbit 5 represents the largest one in Fig. 1. Then, let us denote by $\psi_{i j}$ and $\phi_{i j}$ the spherical coordinates $\psi$ and $\phi$ for the deputy vehicle with respect to the chief satellite, placed along the orbit $i$, and maintaining the formation $j$. Thus, Fig. 11 shows the angles $\psi_{i j}$ and $\phi_{i j}$ for $i=1,2,3,4,5$ and $j=1,2,3,4$, along the long quasi-periodic orbits. As can be seen in Fig. 11, for formations near $L_{4}$, the cost estimate for keeping a formation along the ZRRA cones increases when the coordinate $\psi$ points out in the same direction that the vector that connects the Earth's center with $L_{4}$, i.e., $\psi$ about 60 degrees (or 240 degrees). In addition, the cost decreases when the coordinate $\psi$ points out in the direction orthogonal to the vector that connects the Earth's center with $L_{4}$, i.e., $\psi$ about 150 degrees (or 330 degrees). Additionally, Fig. 11, shows that the vertical component of formations 3 and 4 remains much closer to the $(x, y)$-plane $(\varphi$ about -5 degrees) than formations 1 and 2 ( $\varphi$ about -60 degrees). The orientation of the four formations are illustrated in Fig. 12. It is worth mentioning that, similar results regarding the orientation of the formation, were found by Salazar et al. (2014) for planar formations near $L_{4}$ in the Earth-Moon CRTBP scenario.

In the next section, the influence of the Sun's gravitational attraction in a formation flying about equilateral libration points of the CRTBP will be studied.

\section{Minimum Relative Acceleration Lines in a family of periodic solutions about $L_{4}$}

\subsection{The Sun-Earth-Moon BCFBP scenario}

In this section, we will determine the regions with Zero Relative Radial Acceleration component for the family of periodic orbits about $L_{4}$ shown in Fig. 3, Fig. 4, and Fig. 5. Since the periodic orbits lie on the orbital plane, only planar formations will be considered as a first approximation. Thus, the matrix that must be analyzed along the planar trajectories corresponds to the sub-matrix of $F$, composed by the partial derivatives of the pseudo potential $\Omega^{S}$ with respect to $x$ and $y$.

Hence, let us define matrix $F_{x y}$ as

$$
F_{x y}=\left(\begin{array}{cc}
\Omega_{x x}^{S} & \Omega_{x y}^{S} \\
\Omega_{y x}^{S} & \Omega_{y y}^{S}
\end{array}\right),
$$

Similarly, since matrix $F_{x y}$ is symmetric, it can be diagonalized and Eq.(15) can be rewritten for the planar case in the following form

$$
\lambda_{1} \delta \eta_{1}^{2}+\lambda_{2} \delta \eta_{2}^{2}=0,
$$



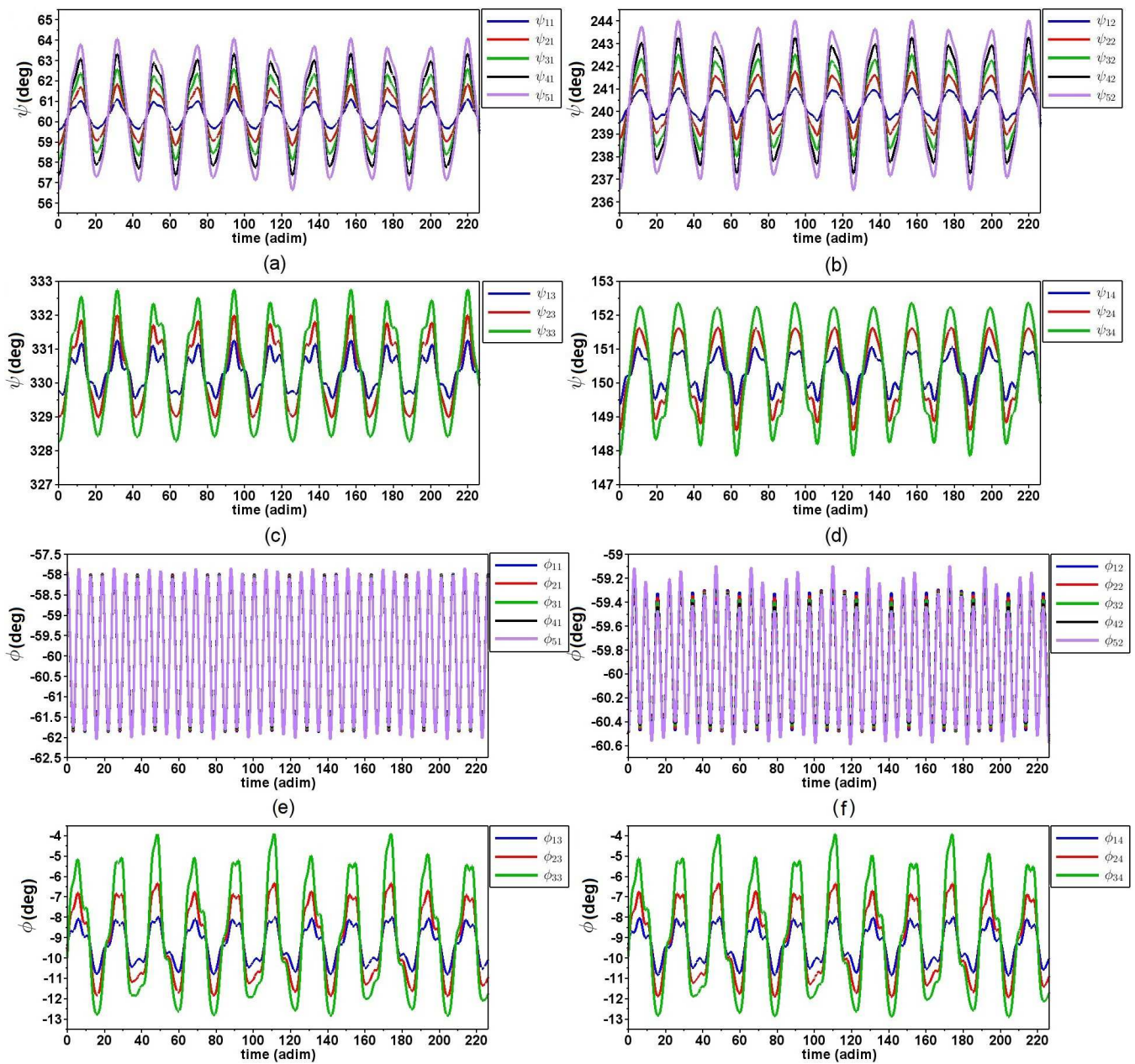

(g)

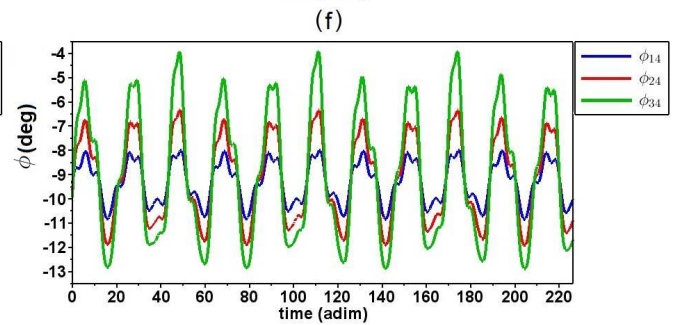

(h)

Figure 11: (a)-(h) Spherical coordinate $\psi_{i j}$ and $\phi_{i j}$ for the deputy vehicle with respect to the chief satellite, placed along the long quasi-periodic orbit $i$, maintaining the formation $j$, during 226 units of dimensionless time (about 1,000 days).

where $\lambda_{1}$ and $\lambda_{2}$ denotes the eigenvalues of matrix $F_{x y}$. Eq.(25) represents a curve of the second order and there are 9 standard-form types (Lawrence, 1972; Barry, 2007). However, since the right side of Eq.(21) is equal to zero, then there are only one type of quadratic curve to be investigated: the intersecting lines. To determine the existence of intersecting lines in Eq.(25), the sign of the product between the eigenvalues $\lambda_{1}, \lambda_{2}$ must be examined. Thus, if the sign of the product is negative, then the curves described by Eq.(25) are intersecting lines, otherwise there does not exist any curve. In this investigation only the real domain in Eq.(25) is examined. 


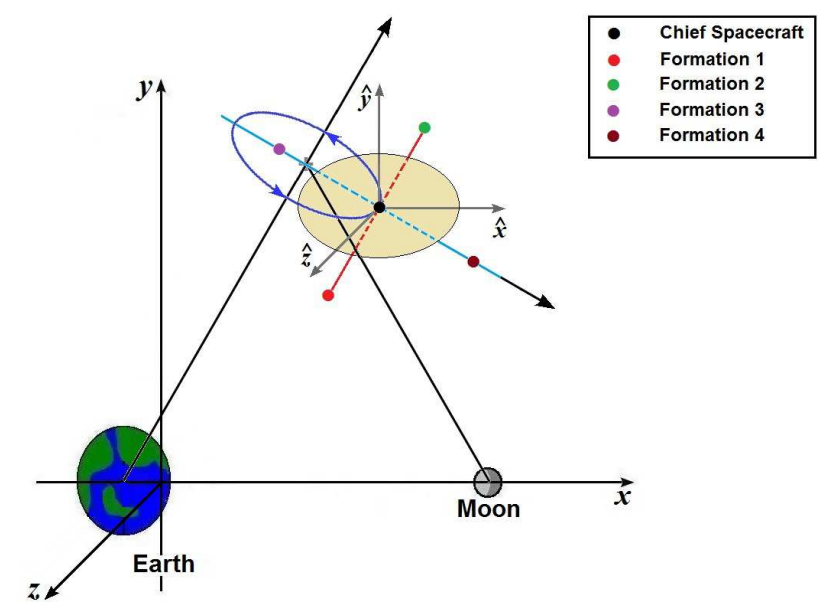

Figure 12: Illustration of the orientation with respect to the $(x, y, z)$ space of each formation, defined in Fig. 10, along the quasi-periodic orbits shown in Fig. 1 (not to scale).

Thus, the existence of intersecting lines depends on the given reference orbit about $L_{4}$ and the corresponding properties of sub-matrix $F_{x y}$.

Fig. 13 shows the eigenvalues $\lambda_{1}$ and $\lambda_{2}$ along the periodic orbits shown in Fig. 3, Fig. 4, and Fig. 5. As can be seen in Fig. 13, since eigenvalue $\lambda_{2}$ is always positive along all five periodic orbits, then the sign of the product between the eigenvalues depends exclusively on the sign of eigenvalue $\lambda_{1}$. Examining the sign of $\lambda_{1}$, Fig. 13 shows that $\lambda_{1}$ is negative uniquely in certain intervals, i.e., there exist only some locations with Zero Relative Radial Acceleration component along these periodic solutions. Therefore, at these positions, the product of the eigenvalues of the sub-matrix $F_{x y}$ is negative, and the quadratic curve becomes intersecting lines at these particular locations (see Fig. 14). In particular, the lower part of all five reference periodic orbits, as presented in Fig. 14, is in closest proximity with the Earth, leading to a higher level of sensitivity in the vicinity of these reference paths. As a consequence, no regions of zero drift are analytically indicated outside of this portion of the orbits.

The previous fact can also be illustrated considering a sphere of radius $2 \mathrm{~km}$ (a circle of $2 \mathrm{~km}$ in the planar case) defined previously in Fig. 7, and computing numerically the radial component of the relative acceleration at two different locations along the periodic orbits. The qualitative behavior of the radial acceleration function is almost the same for all the locations along the periodic orbits $A, B, C, D$, or $E$. Two specific locations, identified as $t=2.037$ and $t=5.432$ units of dimensionless time (about 9 days and 24 days) along the reference paths, are selected inside the 

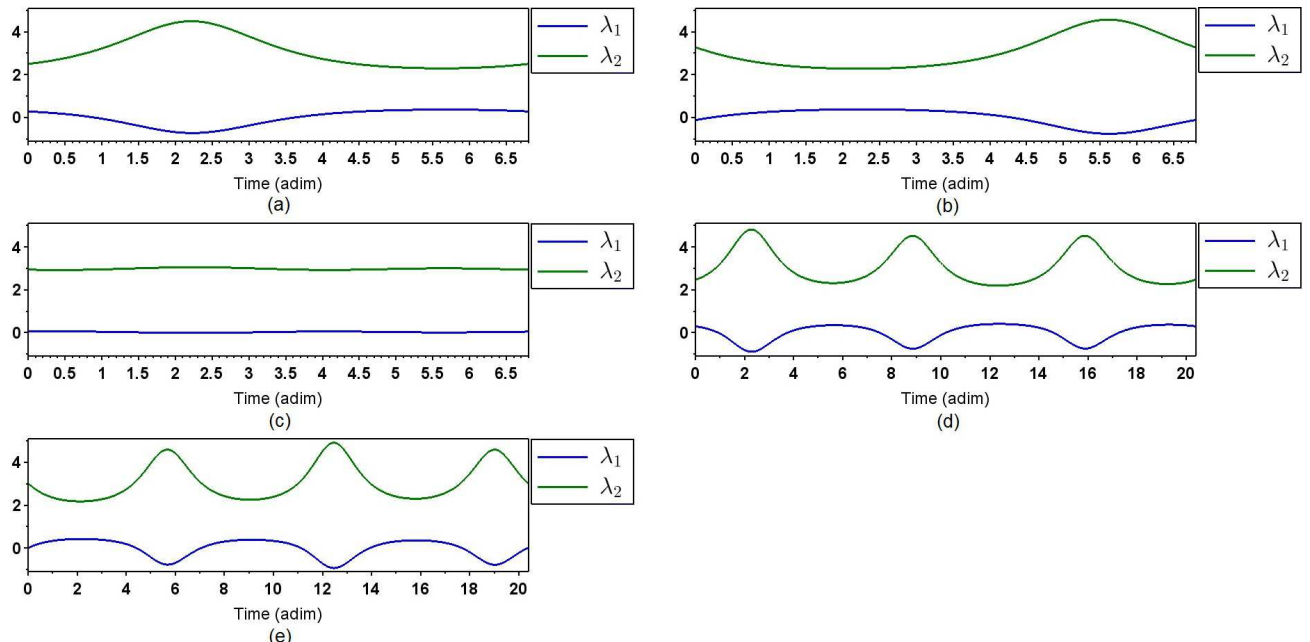

(d)

Figure 13: (a)-(e) Eigenvalues $\lambda_{1}$ and $\lambda_{2}$ along the periodic orbits $A, B, C, D, E$, shown in Fig. 3, Fig. 4, and Fig. 5.
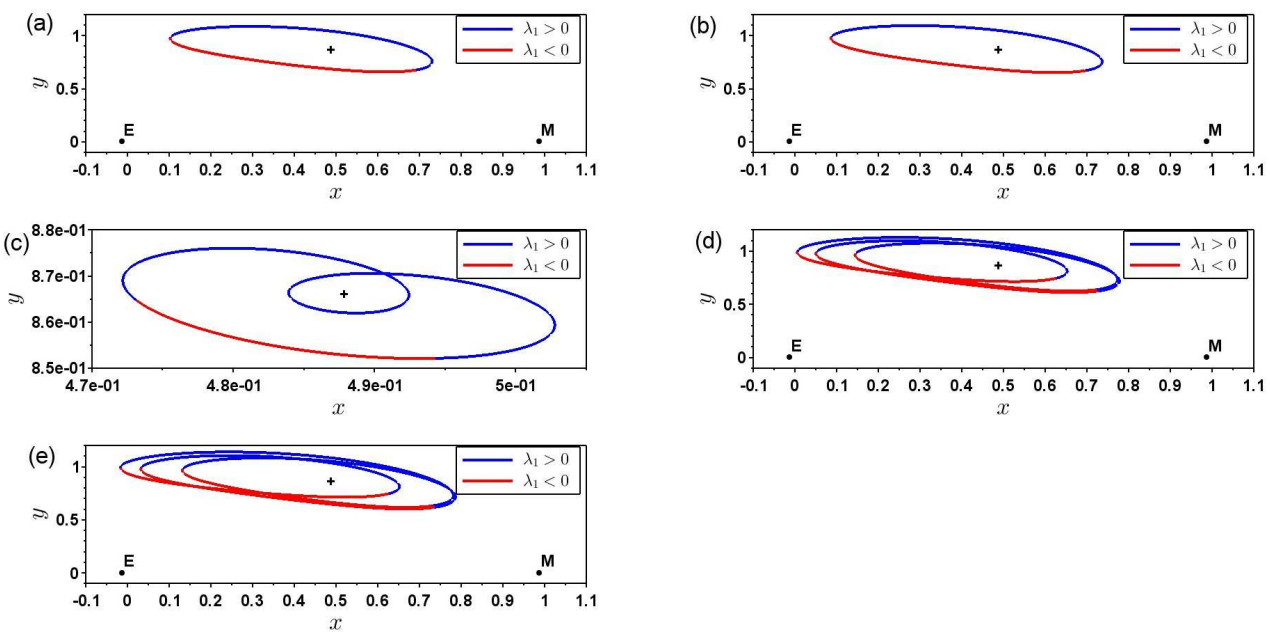

Figure 14: (a)-(e) Regions with Zero Relative Acceleration component $\left(\lambda_{1}<0\right)$, along the periodic orbits $A, B, C, D, E$, shown in Fig. 3, Fig. 4, and Fig. 5.

regions with zero relative radial acceleration component. The radial component of the relative acceleration is then plotted as a function of the angle $\psi$ and represented in Fig. 15. A set of test points on the sphere of radius $2 \mathrm{~km}$ is defined around this location, varying the angle $\psi$ between 0 and 360 degrees, with $\phi=0$ degrees (see Fig. 7). The relative radial acceleration component is evaluated for each of these points using the right side of Eq.(4). This function is periodic (period equal 
to 180 degrees) and has two maxima and two minima similarly to the elliptic case. Additionally, there exist two different values of $\psi$, denoted by $\psi^{*}$ and $\psi^{* *}$, such that, $0<\psi^{*}<\psi^{* *}<200$ degrees, where the radial component is zero with vertex at these locations. Note that since the radial component function is periodic with respect to $\psi$, the other two zeros represent the same situation. In locations where there is no zero drift curves, the radial component function only has two maxima and two minima, and never approaches to zero for any value of $\psi$ at this location.
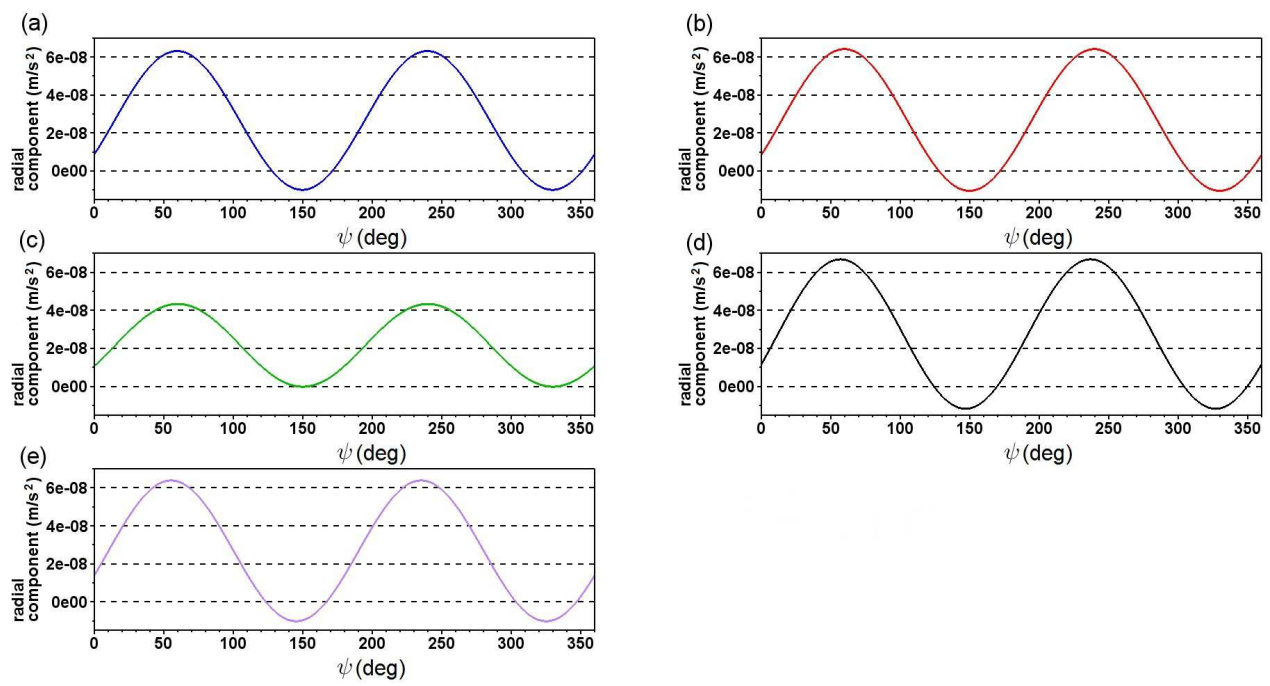

Figure 15: (a)-(e) Radial component of the relative acceleration as a function of angle $\psi$ at one specific location identified as $t=2.037,5.432$ units of dimensionless time (about 9 days and 24 days) along the periodic orbits $A, B, C, D$, and $E$.

\subsection{Cost Estimate to maintain a formation on the Minimum Relative Radial Accel- eration component}

Considering a separation between the deputy and chief spacecraft of $2 \mathrm{~km}$ and only one geometrical configuration, the cost estimate to maintain a formation moving within the Minimum Relative Radial Acceleration (MRRA) component is computed in this section. Similarly, we will assume that the formation has only three spacecraft: two deputy vehicles at the edges of a segment parametrized by the angle $\psi$ (see Fig. 7) and the chief satellite at the middle point. The third satellite will move along the periodic orbits shown in Fig. 3, Fig. 4, and Fig. 5 without any control acting on it. The length of the segment will then be $4 \mathrm{~km}$.

Previous section showed that at certain locations along the periodic orbits $A, B$, $C, D$ and $E$, a planar formation can not be set at the directions of zero drift. The 
curves obtained with the analytical linear approach, and that determines the most suitable directions to set a formation, do not exist along the all locations of the reference paths. On the other hand, the numerical approach showed the existence of a minimum value, denoted by $\psi_{m}$, of the radial component function at the specific locations, such that, $0<\psi_{m}<180$ degrees (Fig. 15). Fig. 16 shows the two zeros $\psi^{*}$, $\psi^{* *}$ and the minimum $\psi_{m}$ associated to each point $X_{h}(t)$ along the reference paths maintaining a fixed distance of $2 \mathrm{~km}$. As can be seen in Fig. 16, the function $\psi_{m}$, which describes the direction of minimum radial component, is smooth. Nevertheless, when the formations are set at the directions where the radial component is minimum or zero, there are two points where the function $\psi$ is not smooth. This fact will imply a discontinuity in the velocity and acceleration components of the relative position vector $\delta r_{a}$ and therefore, a higher cost to maintain a rigid formation about $L_{4}$, as shown by Salazar et al. (2014) in the Earth-Moon CRTBP scenario. Hence, considering only the MRRA that ideally determines low drift regions, the deputy spacecraft will be set at the directions given by the angle $\psi_{m}$.

The relative position vector $\delta r_{a}$ of each deputy satellite from the initial location along the reference orbit during 1,000 days will be computed by transformation of polar coordinates. Similarly, the first and second derivatives of the coordinates of vector $\delta r_{a}$ with respect to time will be approximated by a central finite difference of eighth-order accuracy. Finally, the coordinates of residual acceleration vector $R_{a}$ will be computed using Eq.(16) and the cost estimate $\Delta V$ to maintain the formation within the MRRA curves during 1,000 days will be determined numerically integrating the expression defined in Eq.(17) by the Trapezoid method.

Table 3 shows the cost estimate $\Delta V(\mathrm{~m} / \mathrm{s})$ for the geometrical configuration that maintains two deputy spacecraft within the MRRA curves defined along the periodic orbits $A, B, C, D$, and $E$, during 1,000 days, and keeping a fixed distance of $2 \mathrm{~km}$ between the deputy and chief spacecraft. The first deputy vehicle is set at $\psi=\psi_{m}$, and the second one at $\psi=\psi_{m}+180$ degrees. Note that, for stable periodic orbits $A$ and $B$, and unstable periodic orbits $D$ and $E$, the cost estimate practically remains about $0.5 \mathrm{~m} / \mathrm{s}$, whereas the lowest cost estimate is obtained for periodic orbit $C$, which is also unstable, has the same period of orbits $A$ and $B$, but do not lie far away from $L_{4}$. Therefore, for the periodic orbits studied in this work, the cost estimate depends neither on their period nor on their stability, it depends on their amplitude of the orbit. Large amplitude implies a higher cost estimate to keep the formation.

Similarly, for periodic orbits $A, B, D$, and $E$, angle $\psi_{m}$ varies between 130 degrees and 180 degrees, while on the contrary, for periodic orbit $C, \psi_{m}$ is practically equal to 150 degrees, i.e., the formation in this case points out in the direction orthogonal 

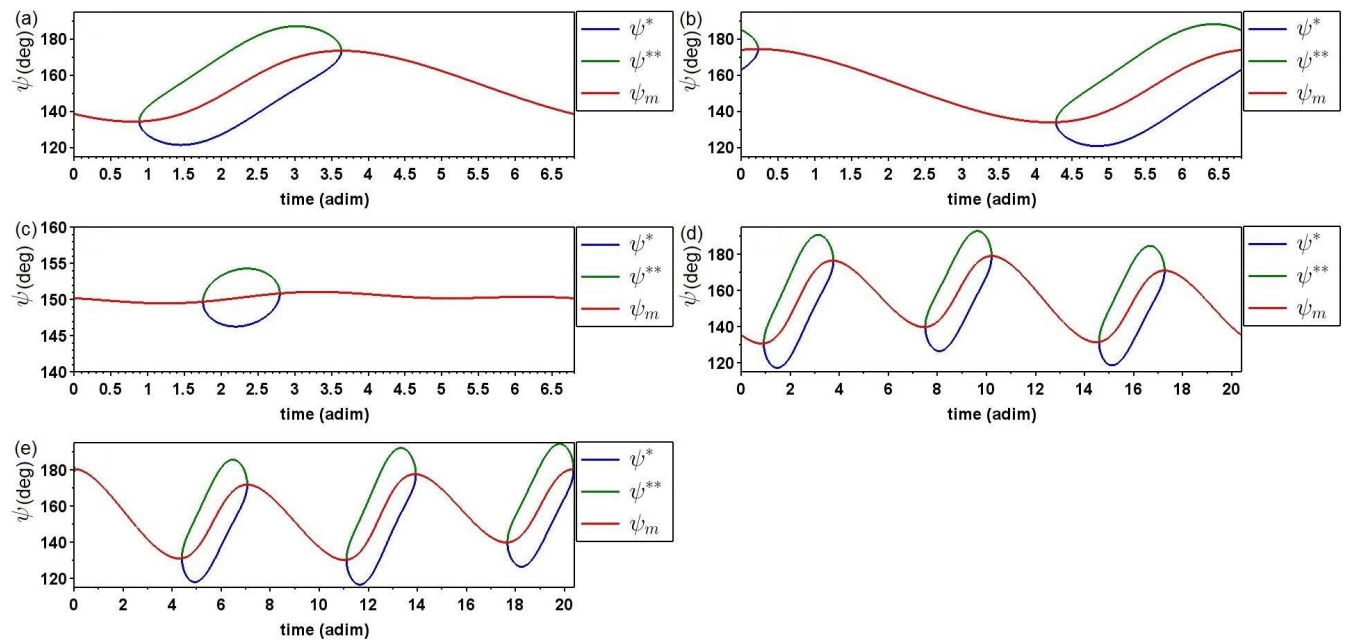

Figure 16: (a)-(e) Zeros $\psi^{*}, \psi^{* *}$ and the minimum $\psi_{m}$ associated to each point $X_{h}(t)$ along the periodic orbits $A, B, C, D$, and $E$, maintaining a distance of $2 \mathrm{~km}$.

\begin{tabular}{llll}
\hline Periodic Orbit & Period (days) & Stability & $\Delta V(\mathrm{~m} / \mathrm{s})$ \\
\hline$A$ & 29 & stable & 0.5314 \\
$B$ & 29 & stable & 0.4712 \\
$C$ & 29 & unstable & 0.0686 \\
$D$ & 88 & unstable & 0.5932 \\
$E$ & 88 & unstable & 0.5902 \\
\hline
\end{tabular}

Table 3: Cost estimate $\Delta V(\mathrm{~m} / \mathrm{s})$ to maintain two deputy spacecraft within the MRRA curves defined along the family of periodic orbits $A, B, C, D$, and $E$ shown in Fig. 3, Fig. 4, and Fig. 5, during 1,000 days, and keeping a fixed distance of $2 \mathrm{~km}$ between the deputy and chief spacecraft.

to the vector that connects the Earth's center and $L_{4}$ point. Identical property was found for the family of quasi-periodic orbits near $L_{4}$ studied in the previous sections. The orientation of the formation set at the MRRA regions is illustrated in Fig. 17.

Finally, Table 3 shows that, although the Sun's attraction force affects the stability of equilateral equilibrium points in the Earth-Moon CRTBP, the formation keeping $\Delta V$ requirements are still very small. However, the cost estimates in this section for large amplitude periodic orbits are almost equal to the average costs computed by Wong (2009), using LQR control, for reference in-plane elliptical relative trajectories near the Earth-Moon $L_{4}$ point in the BCFBP scenario. In fact, for a similar formation studied in this investigation, Wong (2009) would require an average change in velocity of $56 \mathrm{~cm} / \mathrm{s}$ during 1,000 days to maintain the spacecraft near $L_{4}$; whereas placing the formation at periodic orbit $C$, which remains close to $L_{4}$, the cost estimate within 

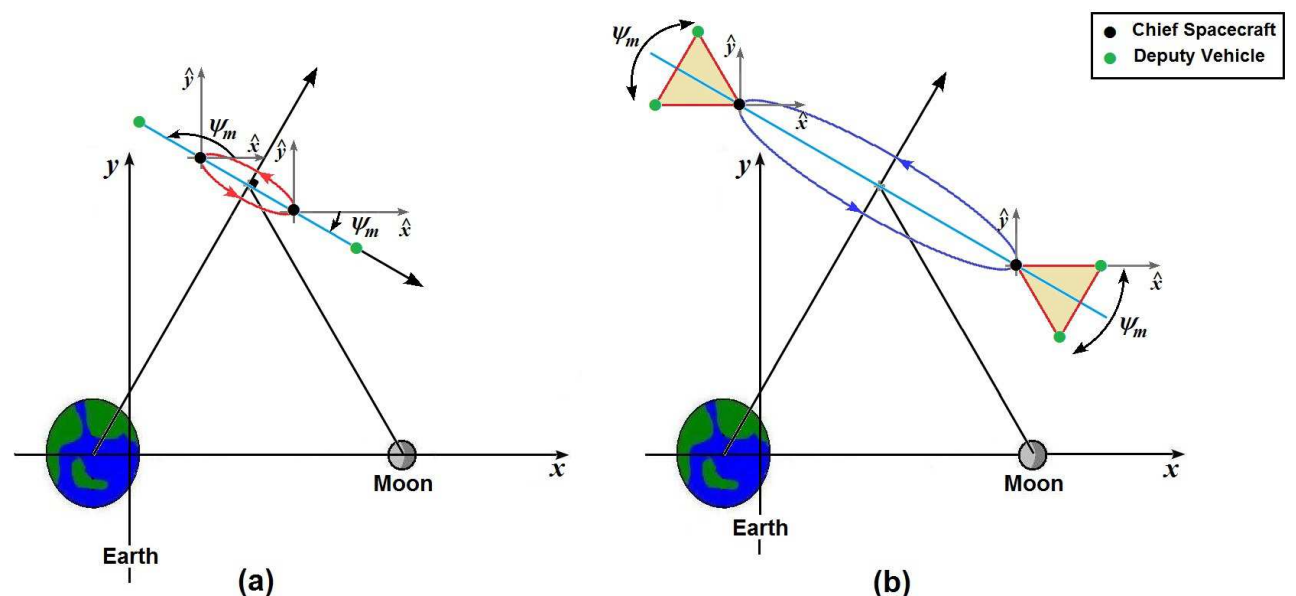

(b)

Figure 17: Illustration of the orientation with respect to the $(x, y)$ space for a formation placed at the MRRA regions, along the periodic orbits near (a) and far (b) from $L_{4}$ in the Earth-Moon BCFBP scenario (not to scale).

the MRRA curves is eight times lower (about $7 \mathrm{~cm} / \mathrm{s}$ ).

\section{Conclusions}

In this paper we have determined quadratic surfaces associated to any natural trajectory suitable for formation flight about the equilateral equilibrium points in two scenarios: Earth-Moon ERTBP and Sun-Earth-Moon BCFBP. In the first scenario, a family of long and short quasi-periodic orbits near $L_{4}$ are considered. Fixing the vertical amplitude, regions with zero relative radial acceleration component for the deputy vehicle are determined varying the long and short amplitudes. Assuming a radius of $2 \mathrm{~km}$ for the geometry of the formation, the variational equation with respect to the reference trajectory derives analytically quadratic surfaces for the zero drift regions along each reference orbit. Although the linear approach determines elliptic cones along the reference paths, from 0.04 adim for the long period and 0.02 adim for the short period amplitude, there exist certain locations where the cones become intersecting planes and their orientation changes. In the second scenario, a family of five planar periodic orbits around $L_{4}$ are considered. For planar formations, the zero drift regions represent intersecting lines, however, these curves only exist for some locations along all five periodic orbits. This fact depends neither on the amplitude nor on the stability of the orbits, but on the amplitude of the periodic solution about $L_{4}$.

The natural motion for the deputy spacecraft, set at the most suitable directions, 
avoids large variations of the mutual distances between the deputy and chief spacecraft. The cost estimate to maintain a certain geometrical configuration along the reference paths, with a fixed radius of $2 \mathrm{~km}$, have been computed by the integral of residual acceleration.

In the ERTBP scenario, four different geometrical configurations in the ZRRA cones are considered. To avoid the indetermination produced by the bifurcation between the elliptic cones and the intersecting planes, the first two configuration remain on the plane generated by the first and third eigenvectors of the matrix of all second-order partial derivatives of the pseudo potential; the last two configurations remain on the plane generated by the first two eigenvectors if there is no indetermination along the reference paths. Even though the first two configuration satisfy the quadratic equation along each quasi-periodic orbit analyzed in this study, a lower cost estimate was found for the last two configurations, which represent quasi-planar formation with respect to the coordinate system parallel to the rotating coordinate system $x, y, z$ and centered at the chief's path, such that, the deputy's relative position vector and the line that connects the Earth's center with $L_{4}$ are practically orthogonal. Planar formations thus appear to be the most robust of the formations designed here.

On the other hand, in the BCFBP scenario, since the zero drift curves does not exist for all locations along the periodic orbits, the planar formation points out in the direction where the radial component of the relative acceleration reaches a minimum. This configuration avoids the discontinuity in the relative velocity and acceleration components. A lower cost estimation is obtained when the formation remains close to $L_{4}$, and similar to the ERTBP scenario, the orientation is orthogonal to the vector that connects Earth's center with $L_{4}$ point.

Although the ERTBP and BCFBP scenarios significantly affect the dynamics about $L_{4}$ point, the formation keeping $\Delta V$ requirements in this work are extremely small, on the order of $10 \mathrm{~cm} / \mathrm{s}$ during 1,000 days. Therefore, for small satellites with a mass of few kilograms, such small velocity changes are achievable using electric propulsion systems.

In conclusion, this research has provided a more accurate description of the relative motion about Earth-Moon triangular libration points because Moon's eccentricity and solar gravitational effects were modeled and added to the system. However, since ERTBP and BCFBP models catch the basic dynamics of formation flight about equilateral libration points in the Earth-Moon system, this analysis presented here consists in a preliminary study to mission design and simulation, and it can be extended to more complex descriptions of relative motion at $L_{4}$ point, e.g., ephemerisbased model. Finally, new reference orbits may be included in different environments, 
in such a way that a more real description of the most suitable directions for formation at triangular libration points could be obtained for potential applications.

\section{Acknowledgments}

Firstly, the authors thanks the financial support of the Fapesp (Fundação de Amparo à Pesquisa do Estado de São Paulo), processes 2008/06066-5, 2011/08171-3, 2011/50151-0, and 2013/03233-6, and the CNPq (Conselho Nacional para Desenvolvimento Científico e Tecnológico, Brazil). E.E.N.M. is supported by FAPESP 2011/50151-0. In the other hand, J. J. M. thanks the MINECO-FEDER grant MTM2012-31714 and the Catalan grant 2014SGR-504. G. G. thanks the MINECOFEDER grant MTM2013-41168-P and the Catalan grant 2014SGR-1145. Finally, the authors are also grateful to profs. H. Lei and B. Xu of the School of Astronomy and Space Science at Nanjing University from China, who provided the coefficients of the third-order series expansion to obtain the family of periodic solutions around the triangular equilibrium point in the elliptic case.

Barry, S. Analytical Conics, Dover Publ., New York, NY., 2007

Battrick, B., 2000. XEUS Steering Committee: X-Ray Evolving-Universe Spectroscopy: The XEUS Mission Summary. ESA Publications Division, Noordwijk, the Netherlands, 2000

Bristow, J., Folta, D., Hartman, K. A formation flying technology vision. AIAA Space Conf., Long Beach, California, 2000. AIAA Paper No. 2000-5194

Burns, R., McLaughlin, C.A., Leitner, J., et al. TechSat 21: formation design, control, and simulation. IEEE Aero. Conf., 2000

Cash, W., Kendrick, S., Noecker, C., et al. The New Worlds Observer: The Astrophysics Strategic Mission Concept Study. Proc. SPIE, Vol. 7436, article id. 743606, 14 pp., 2009. http://dx.doi.org/10.1117/12.827486

Catlin, K.A., McLaughlin, C.A. Earth-Moon Triangular Libration Point Spacecraft Formations. J. Spacecr. Rockets 44 (3), 660-670, 2007. http://dx.doi.org/10.2514/1.20152

Danby, J.M. Stability of the triangular points in the elliptic restricted problem of three bodies. Astron. J. 69(2), 165-172, 1964 
Defilippi, G., Jr. Station keeping at the $L_{4}$ libration point: A three dimensional study. Thesis. School of Engineering, Air Force Inst. of Tech., Wright-Patterson AFB, OH, M.Sc., 1977

Díez, C., Jorba, À., Simó, C. A Dynamical Equivalent to the Equilateral Libration Points of the Earth-Moon Real System. Celest. Mech. 50 (1), 13-29, 1991. http://dx.doi.org/10.1007/BF00048984

Drazin, P.G. Nonlinear systems, Cambridge University Press, Cambrige, UK, 181186, 1992

Érdi, B., Forgács-Dajka, E., Nagy, I., et al. A parametric study of stability and resonances around $L_{4}$ in the elliptic restricted three-body problem. Celest. Mech. Dyn. Astron. 104, 145-158, 2009. http://dx.doi.org/10.1007/s10569-009-9197-2

Faquhar, R. W. The Control and Use of Libration-Point Satellites. Thesis. Dept. of Aeronautics and Astronautics, Stanford University, Ph.D., 1968

Feldt, W. T., Schulman, Y. More results on solar influenced libration point motion. AIAA J. 4 (4), 1501-1502, 1966

Fitzpatrick, R. An introduction to celestial mechanics. Cambridge University Press, New York, NY., 2012

Flick, M. Earth Observing-1: Preliminary Technology and Science Validation Report. Goddard Space Flight Center. http://eo1.gsfc.nasa.gov/new/validationReport/index.html 01 Nov. 2012

Fornberg, B. Generation of Finite Difference Formulas on Arbitrarily Spaced Grids. Math. Comp. 51 (184), 699-706, 1988

Fridlund, C.V.M., Capaccioni, Fabrizio. Infrared space interferometrythe DARWIN mission. Adv. Space Res. 30 (9), 2135-2145, 2002. http://dx.doi.org/10.1016/S0273-1177(02)00585-9

Gill, E., Montenbruck, O., D'Amico, S. Autonomous Formation Flying for the PRISMA Mission. J. Spacecraft Rockets 44 (3), 671-681, 2007. http://dx.doi.org/10.2514/1.23015

Gómez, G., Lo, M., Masdemont, J.J., et al. Simulation of Formation Flight Near $L_{2}$ for the TPF Mission. AAS/AIAA Astro. Specialist Conf., Quebec City, Canada, July 2001. Paper No. AAS 01-305. 
Gómez, G., Llibre, J., Martínez, R., et al. Dynamics and Mission Design Near Libration Point Orbits, Vol. II, Fundamentals: The case of Triangular Libration Points. World Scientific, Singapore, 2001

Gómez, G., Jorba, À., Masdemont, J.J., et al. Dynamics and Mission Design Near Libration Point Orbits, Vol. IV, Advanced Methods for Triangular Points. World Scientific, Singapore, 2001

Gómez, G., Marcote, M., Masdemont, J.J., et al. Natural Configurations and Controlled Motions Suitable for Formation Flying (AAS 05-347). Adv. Astronaut. Sci. 123 (2), 1513-1530, 2006

Hellman, H., Persson, S., Larsson, B. PRISMA, a Formation Flying Mission on the Launch Pad. 60th Int. Astro. Congress, Daejeon, Republic of Korea, Oct. 2009

Héritier, A., and Howell, K. Dynamical Evolution of Natural Formations in Libration Point Orbits in a Multi-Body Regime. Acta Astronaut., 102, 332-340, 2014. http://dx.doi.org/10.1016/j.actaastro.2013.10.017

Hilbert, D., Cohn-Vossen, S. The Second-Order Surfaces, Geometry and Imagination, Vol. 3, 12-19, 1999

Howell, C., Marchand, B.G. Natural and Non-Natural Spacecraft Formations Near the $L_{1}$ and $L_{2}$ Libration Points in the Sun-Earth/Moon Ephemeris System. Dyn. Syst. Int. J. 20 (1), 149-173, 2005

Hsiao, F. Y., Scheeres, D. J. The Dynamics of Formation Flight About A Stable Trajectory. J. Astronaut. Sci. 50 (3), 269-287, 2002

Huang, S.S. Very restricted four body problem, NASA Technical Note D-501, 1960. http://ntrs.nasa.gov/archive/nasa/casi.ntrs.nasa.gov/19890068606.pdf

Jorba, À. A numerical study on the existence of stable motions near the triangular points of the real Earth-Moon system. Astron. Astrophys. 364 (1), 327-338, 2000

Kapila, V., Sparks, A.G., Buffington, J.M., et al. Spacecraft Formation Flying: Dynamics and Control. J. Guid. Control Dyn. 23 (3), 561-564, 2000. http://dx.doi.org/10.2514/2.4567

Kolenkiewicz, R., Carpenter, L. Periodic motion around the triangular libration points in the restricted problem of four bodies. Astron. J. 72 (2), 180-183, 1967 
Kolenkiewicz, R., Carpenter, L. Stable periodic orbits about the Sun perturbed Earth-Moon triangular points. AIAA J. 6 (7), 1301-1304, 1968

Lawrence, J. D. A Catalog of Special Plane Curves, Dover Publ., New York, NY., 1972

Lei, H., Xu, B. High-order solutions around triangular libration points in the elliptic restricted three-body problem and applications to low energy transfers. Commum. Nonlinear Sci. Numer. Simulat. 19 (9), 3374-3398, 2014. http://dx.doi.org/10.1016/j.cnsns.2014.01.019

Marchand, G., Howell, K.C. Control Strategies for Formation Flight in the Vicinity of the Libration Points. J. Guid. Control Dynam. 28 (6), 1210-1219, 2005

Munoz, J.P. Sun-Perturbed Dynamics of a Particle in the Vicinity of the Earth-Moon Triangular Libration Points. Thesis. Dept. of Aerospace Engineering, University of Texas at Austin, Ph.D., 2008

O'Neill, G.K. The Colonization of Space. Phys. Today 27 (9), 32-40, 1974. http://dx.doi.org/10.1063/1.3128863

Perea, L. Design and Evaluation of Navigation and Control Algorithms for Spacecraft Formation Flying Missions. Thesis. Departament de Matemàtica Aplicada i Anàlisi, Universitat de Barcelona, Barcelona, Ph.D., 2006

Persson, S., Bodin P., Gill, E., et al. PRISMA An Autonomous Formation Flying Mission. Proc. Small Satellite Systems and Services Symp., 25-29, Sardinia, Italy, Sept. 2006

Persson, S., D'Amico, S., Harr, J. Flight Results from PRISMA Formation Flying and Rendezvous Demonstration. 61st Int. Astro. Congress, Prague, Czech Republic, Sept. 2010

Peterseim, M., Robertson, D.I., Danzmann, K., et al. LISA interferometer sensitivity to spacecraft motion. Adv. Space Res. 25 (6), 1143-1147, 2000. http://dx.doi.org/10.1016/S0273-1177(99)00975-8

Press, W. H., Teukolsky, S. A., Vetterling, W. T., et al. Numerical recipes in C: The art of scientic computing, 2nd ed. Cambridge University Press, Cambridge, 1992

Sabol, C., Burns, R., McLaughlin, C.A. Satellite Formation Flying Design and Evolution. J. Spacecraft and Rockets 38 (2), 270-278, 2001. http://dx.doi.org/10.2514/2.3681 
Salazar, F., Masdemont, J.J., Gómez, G., et al. Zero, Minimum and Maximum Relative Radial Acceleration for Planar Formation Flight Dynamics near Triangular Libration Points in the Earth-Moon System. Adv. Space Res. 54 (9), 1838-1857, 2014. http://dx.doi.org/10.1016/j.asr.2014.07.018

Schechter, H.B. Three-Dimensional Nonlinear Stability Analysis of the SunPerturbed Earth-Moon Equilateral Points, AIAA J. 6 (6), 1223-1228, 1968

Schutz, B.E. Orbital Mechanics of Space Colonies at $L_{4}$ and $L_{5}$ of the Earth-Moon System. AIAA Astro. Specialist Conf., Los Angeles, CA., Jan. 1977. AIAA Paper No. 77-33

Simó, C., Martínez, R., Gómez, G., et al. Relative motion near the Triangular Libration Points in the Earth-Moon system. ESA Workshop on Optical Interferometry in Space, Granada, Spain, Aug. 1987. ESA SP-273

Simó, C., Gómez, G., Jorba, À., et al. The bicircular model near the triangular libration points of the RTBP. In: From Newton to Chaos, 343-370, 1995

Sholomitsky, G.B., Prilutsky, O.F., Rodin, V.G. Infra-red space interferometer. 28th Int. Astro. Fed. Congress, Praha, Czechoslovakia, 1977. Paper IAF-77-68

Stoer, J., Bulirsch, R. Introduction to numerical analysis, 1st ed. Springer Verlag, New York, NY., 1980

Szebehely, V. Theory of Orbits. Academic Press Inc., New York, NY, 1967

Tapley, B. D., Lewallen, J.M. Solar Influence on Satellite Motion Near the Stable Earth-Moon Libration Points, AIAA J. 2 (4), 728-732, 1964

Tapley, B. D., Schultz, B. E. Further results on solar influenced libration point motion. AIAA J. 3 (10), 1954-1956, 1965

Tapley, B. D., Schultz, B. E. Persistent solar influenced libration point motion, AIAA J. 6 (7), 1405-1406, 1968

Ticker, R.L., Azzolini, J.D. 2000 Survey of Distributed Spacecraft Technologies and Architectures for NASA's Earth Science Enterprise in the 2010-2015 Timeframe. NASA/TM-2000-209964, Aug. 2000

Wolaver, L. E. Effect of initial configurations in libration point motion, AIAA/ION Astro. Specialist Conf., Monterey, California, Sep. 1965. AIAA Paper No. 65-684. 
Wong, F.Y.W. Formation-Keeping Strategies At The Earth-Moon $L_{4}$ Triangular Libration Point. Thesis. Department of Aerospace Engineering, Ryerson University, Toronto, Ontario, Canada, M.A.Sc., 2009 